\title{
Study of the vertical structure of Saturn's atmosphere using HST/WFPC2 images
}

\author{
O. Muñoz,${ }^{\mathrm{a}, *}$ F. Moreno, ${ }^{\mathrm{a}}$ A. Molina, ${ }^{\mathrm{a}, \mathrm{b}}$ D. Grodent, ${ }^{\mathrm{c}}$ J.C. Gérard, ${ }^{\mathrm{c}}$ and V. Dols ${ }^{\mathrm{c}}$ \\ a Instituto de Astrofísica de Andalucía, CSIC, P.O. Box 3004, 18008, Granada, Spain \\ ${ }^{\mathrm{b}}$ Departamento de Física Aplicada, Universidad de Granada, Granada, Spain \\ ${ }^{\mathrm{c}}$ Laboratory for Planetary and Atmospheric Physics, Université de Liège, Liège, Belgium
}

Received 25 February 2003; revised 3 November 2003

Available online 6 March 2004

\begin{abstract}
We have studied the vertical structure of hazes at six different latitudes $\left(-60^{\circ},-50^{\circ},-30^{\circ},-10^{\circ},+30^{\circ}\right.$, and $\left.+50^{\circ}\right)$ on Saturn's atmosphere. For that purpose we have compared the results of our forward radiative transfer model to limb-to-limb reflectivity scans at four different wavelengths $(230,275,673.2$, and $893 \mathrm{~nm})$. The images were obtained with the Hubble Space Telescope Wide Field Planetary Camera 2 in September 1997, during fall on Saturn's northern hemisphere. The spatial distribution of particles appears to be very variable with latitude both in the stratosphere and troposphere. For the latitude range $+50^{\circ}$ to $-50^{\circ}$, an atmospheric structure consisting of a stratospheric haze and a tropospheric haze interspersed by clear gas regions has been found adequate to explain the center to limb reflectivities at the different wavelengths. This atmospheric structure has been previously used by Ortiz et al. (1996, Icarus 119, 53-66) and Stam et al. (2001, Icarus 152, 407-422). In this work the top of the tropospheric haze is found to be higher at the southern latitudes than at northern latitudes. This hemispherical asymmetry seems to be related to seasonal effects. Different latitudes experience different amount of solar insolation that can affect the atmospheric structure as the season varies with time. The haze optical thickness is largest (about 30 at $673.2 \mathrm{~nm}$ ) at latitudes \pm 50 and -10 degrees, and smallest (about 18) at \pm 30 degrees. The stratospheric haze is found to be optically thin at all studied latitudes from -50 to +50 degrees being maximum at $-10^{\circ}(\tau=0.033)$. At $-60^{\circ}$ latitude, where the UV images show a strong darkening compared to other regions on the planet, the cloud structure is remarkably different when compared to the other latitudes. Here, aerosol and gas are found to be uniformly mixed down to the 400 mbar level.
\end{abstract}

(C) 2004 Elsevier Inc. All rights reserved.

Keywords: Saturn atmosphere; Atmospheres structure; Radiative transfer

\section{Introduction}

Previous studies of the cloud structure in Saturn atmosphere have revealed significant north/south asymmetries (see, e.g., West et al., 1982, 1983b; Wagener and Caldwell, 1988; Barnet et al., 1992a; Ortiz et al., 1995; Stam et al., 2001). Barnet et al. (1992a) studied the effects of seasonal insolation variations on the thermal response of the atmosphere of Saturn at the time of the Voyager spacecraft encounters. These authors found an enhancement of hydrocarbons in the upper southern stratosphere probably produced by photolysis during Saturn's summer season. In contrast, the northern hemisphere appeared as depleted in

\footnotetext{
* Corresponding author.

E-mail address: olga@iaa.es (O. Muñoz).
}

aerosols after the long winter season. Approximately at the same Saturn's year, West et al. (1982) found cloudy material located at higher altitudes in the northern hemisphere. West et al. (1982) suggested sublimation of high altitude ammonia crystals in the summer (southern) hemisphere. The observed brightness variations seem to be due to differences in the physical properties of the cloud structure, such as their location in the atmosphere and their optical depths. These differences are related to seasonal changes. Dynamical differences are another possible source of the north/south asymmetry because the dynamics is also influenced by different amounts of solar insolation as the season varies with time.

Knowledge of the physical properties of the cloud particles and their location in the atmosphere is of main importance for our understanding of the dynamics of the atmosphere and the influence of seasonal changes on these 
clouds. Since Saturn has an orbital period of 29.48 years, we need to study its atmosphere covering a broad temporal range to shed some light on the relation between seasonal changes and the physical properties of the atmospheric particles. Even though in this work we study images of only two consecutive nights we can search for seasonal effects by comparing the cloud structure of the two hemispheres.

We have studied images taken with the Wide Field Planetary Camera (WFPC2) on board the Hubble Space Telescope (HST). The images were taken on September 10th and 11th 1997, during fall on the northern hemisphere. To constrain as much as possible the vertical distribution and physical properties of the particles in the atmosphere, we have used images covering a wide wavelength range from the UV to the near infrared including a strong methane absorption band. Specifically, we used filters F218W, F255W, F673N, and FQCH4P15 with effective wavelengths, $\lambda_{\text {eff: }}$ : $230,275,673.2$, and $893 \mathrm{~nm}$, respectively. In this way, we cover a large spectral range with the most appropriate wavelengths to sound atmospheric levels from the stratosphere to the troposphere. Our observations extend well into the near UV, where just a few photometric analyses in terms of atmospheric models have been performed so far (see, e.g., West et al., 1983a; Karkoschka and Tomasko, 1993). The observations in the ultraviolet are generally limited to stratospheric levels by Rayleigh scattering. Taking into account the Rayleigh scattering at the two UV wavelengths, the pressure at which the optical depth is equal to one at $230 \mathrm{~nm}$ is 88 mbar whereas at $275 \mathrm{~nm}$ is 193.4 mbar. Therefore, the $230 \mathrm{~nm}$ filter is much more sensitive to upper levels in the atmosphere than the $275 \mathrm{~nm}$ filter. The observations in the methane band at $893 \mathrm{~nm}$ are also limited to high atmospheric levels by methane absorption, reaching an optical depth of one at about 250 mbar. We have included also a continuum wavelength $(673.2 \mathrm{~nm})$ to sound deeper levels into the atmosphere. In absence of aerosols the absorption of radiation at the continuum wavelength $(673.2 \mathrm{~nm})$ is practically negligible. The Rayleigh scattering optical depth at that wavelength is very small and there is no gas absorption. Thus, in principle we could sound the deepest layers in the atmosphere.

To retrieve the vertical profile of clouds we have used an adapted version of the forward radiative transfer model that previously has been used successfully to study the vertical cloud structure of Jupiter's atmosphere (Moreno et al., 1995; Molina et al., 1997; Muñoz et al., 1999). We have considered limb-to-limb reflectivity scans in both hemispheres.
Reflectivity data were extracted and averaged over one degree in latitude for all images. In order to look for possible seasonal effects we have compared our results for the southern (spring) hemisphere (at $-60^{\circ},-50^{\circ},-30^{\circ},-10^{\circ}$ planetocentric latitudes) with those for the northern (fall) hemisphere $\left(+30^{\circ},+50^{\circ}\right.$ planetocentric latitudes $)$. The results of this work are also compared with the results obtained by other authors at different dates: West et al. (1983a); Karkoschka and Tomasko (1993); Ortiz et al. (1996); and Stam et al. (2001).

The description of the observations together with a description of the reduction technique is presented in Section 2. In Section 3, we describe the radiative transfer model and the fitting procedure. Results are presented in Section 4. Discussion and summary are given in Section 5.

\section{Observations and data reduction}

The observational data used in this work consist of $4 \mathrm{im}-$ ages of Saturn taken from the observing program GO-6648 (P.I. J.C. Gérard) with the Planetary Camera (PC) mode of the Wide Field Planetary Camera 2 (WFPC2) on board the Hubble Space Telescope (HST). The images were taken with different filters on September 10 and 11, 1997. The phase angle at the time of the observations was 3.3 degrees. In Table 1, we present the main characteristics of the observations.

The standard Space Telescope Science Institute (STScI) data reduction pipeline procedure has been applied to the images. In addition, we have applied geometric distortion corrections, using the formulae and parameters by Holtzman et al. (1995). Cosmic ray traces are readily identified by the deviation from the median of neighboring pixel values. They were rejected from the images and replaced by the median value.

The reflectivity, $I / F$, where $I$ is the intensity and $\pi F$ is the incident solar flux, has been calculated using flux calibrations for each filter (Karkoschka, 1998a). The conversion factors from counts to reflectivity were corrected for heliocentric distance and adjusted to a PC gain of 7. They also account for the exposure time and the contamination level of the PC camera. Photometric errors come from essentially three sources, namely error in the solar flux, the filter plus system response function, and the error associated with the derivation of the count rates for different geometrical observing conditions. The error in the solar flux is

Table 1

Main characteristics of the observations: date of the observations, time of observations (UTC), filters, effective wavelength when convolved with the solar spectrum $\left(\lambda_{\text {eff }}\right)$ in nm, exposure time (Exp.), and Sub-Earth (SE) and Sub-solar (Ss) planetocentric latitudes and longitudes (system III) in degrees

\begin{tabular}{|c|c|c|c|c|c|c|c|c|c|}
\hline Image & Date $(\mathrm{d} / \mathrm{m} / \mathrm{y})$ & UTC & Filter & $\lambda_{\text {eff }}(\mathrm{nm})$ & Exp. (s) & SE lat. & Ss lat. & SE lon. & Ss lon. \\
\hline U3JJ5A05R & $10 / 9 / 97$ & $17: 41: 13$ & FQCH4P15 & 893 & 80 & -10.95 & -9.84 & 264.74 & 267.75 \\
\hline U3JJ5A0AR & $10 / 9 / 97$ & $20: 56: 13$ & F673N & 673.2 & 10 & -10.94 & -9.84 & 14.54 & 17.55 \\
\hline U3JJ5201R & $11 / 9 / 97$ & $02: 40: 13$ & F $218 W$ & 230 & 800 & -10.94 & -9.85 & 208.25 & 211.26 \\
\hline U3JJ5203R & $11 / 9 / 97$ & $03: 13: 13$ & F255W & 275 & 300 & -10.94 & -9.85 & 226.84 & 229.85 \\
\hline
\end{tabular}




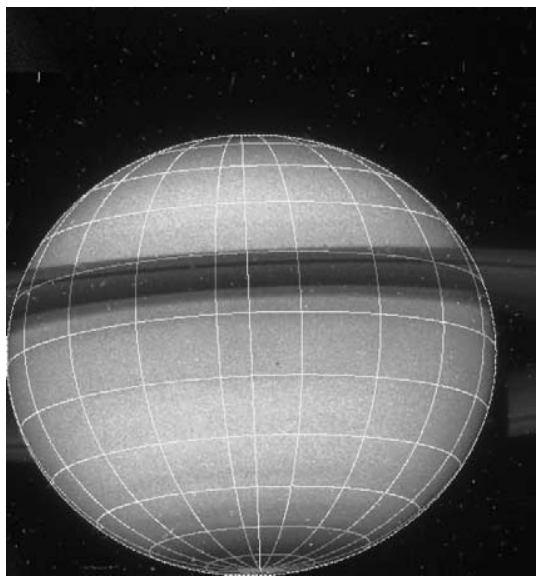

(a)

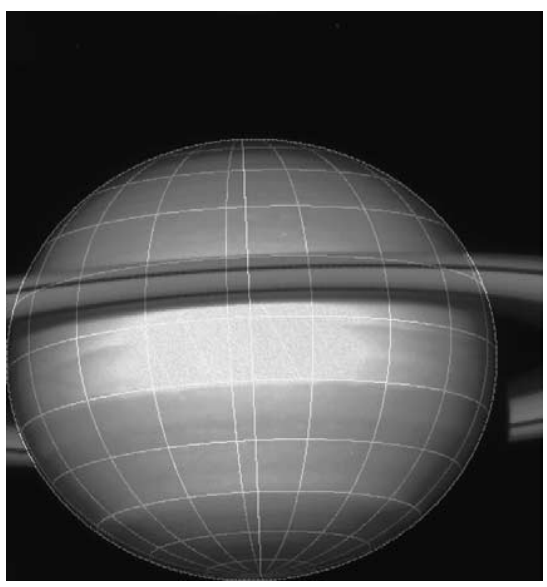

(c)

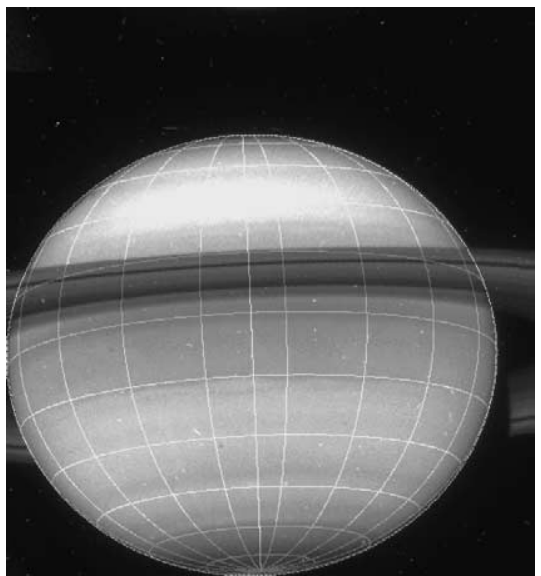

(b)

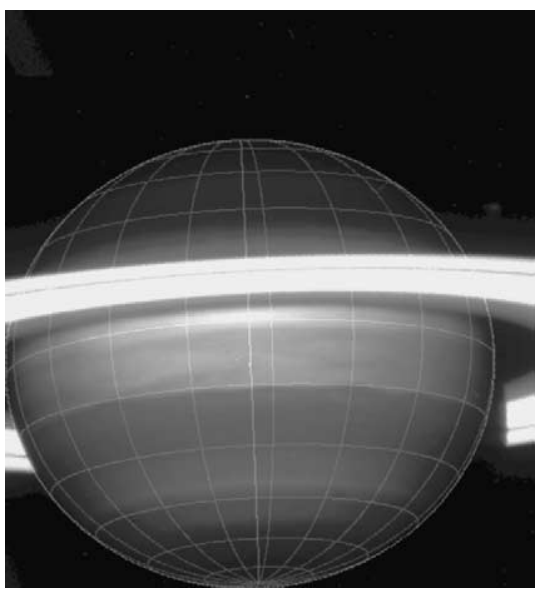

(d)

Fig. 1. Images of Saturn (a) at $230 \mathrm{~nm}$, (b) $275 \mathrm{~nm}$, (c) $673.2 \mathrm{~nm}$, and (d) $893 \mathrm{~nm}$. The images are presented with an overplotted grid $15^{\circ}$ spaced parallels and $20^{\circ}$ spaced meridians.

accurate to 1\% (Karkoschka, 1998a). The error in the filter plus system response function is estimated to be about 3\% (Karkoschka, 1998a). The differences in the geometrical conditions are due to differences in the phase angle and ring parameters $B$ and $B^{\prime}$ (the saturnicentric latitudes of the Earth and the Sun with respect to the ring plane). The count rates obtained by Karkoschka (1998a), were obtained from spectra by Karkoschka (1998b) longward of $300 \mathrm{~nm}$ and geometrical albedo spectra by Wallace et al. (1972), at shorter wavelengths. These spectra were taken at phase angles of 5.7 and 6.3 degrees, respectively. In order to get the count rates at zero phase, Karkoschka (1998a), multiplied the albedos by an estimated factor of 1.05 . Since our observations were made at a phase angle of 3.3 degrees, and in addition, the ring parameters $B$ and $B^{\prime}$ varied among these observations, we estimate a $6 \%$ error associated to the different geometrical conditions. Moreover, with the FQCH4P15 filter a substantial portion of the aperture is affected by vignetting. In order to minimize the effects of vignetting in the FQCH4P15 aperture, the center of Saturn was requested to be placed at the optimum center of the aperture (PC camera + FQCH4P15 filter). In this configuration, most of the Saturn disk appears in the unvignetted portion of the field of view, since only the regions poleward of -70 degrees of latitude are at or beyond the limit of the unvignetted zone. Therefore, the effects of vignetting in the 893 methane filter can be considered negligible. In summary, all inaccuracies in the photometric calibration lead to an upper limit of the error on the absolute $I / F$ values of $10 \%$.

In Fig. 1, we present the four reduced images that have been considered in this study. For each geometrically corrected image, the points of inflection along radial scans around the limb were adjusted to the edge of a model disk accounting for the sub-Earth latitude and the north pole position angle. For each filter, the model disk was convolved by a model Point Spread Function (PSF) generated by the Tiny Tim software package available from the STScI. With this method, the accuracy of the centering of the Saturn disk is \pm 1 pixel. After the disk center is found, the location of the sub-Earth and sub-solar points are entered in a FORTRAN code (developed at the Laboratory for Planetary and Atmospheric Physics, Université de Liège) using the SPICE-NAIF navigation library (provided by the Jet Propulsion Laboratory), to calculate the longitude and latitude coordinates of each pixel of Saturn. Sub-solar and subEarth planetocentric latitudes and longitudes are also given 

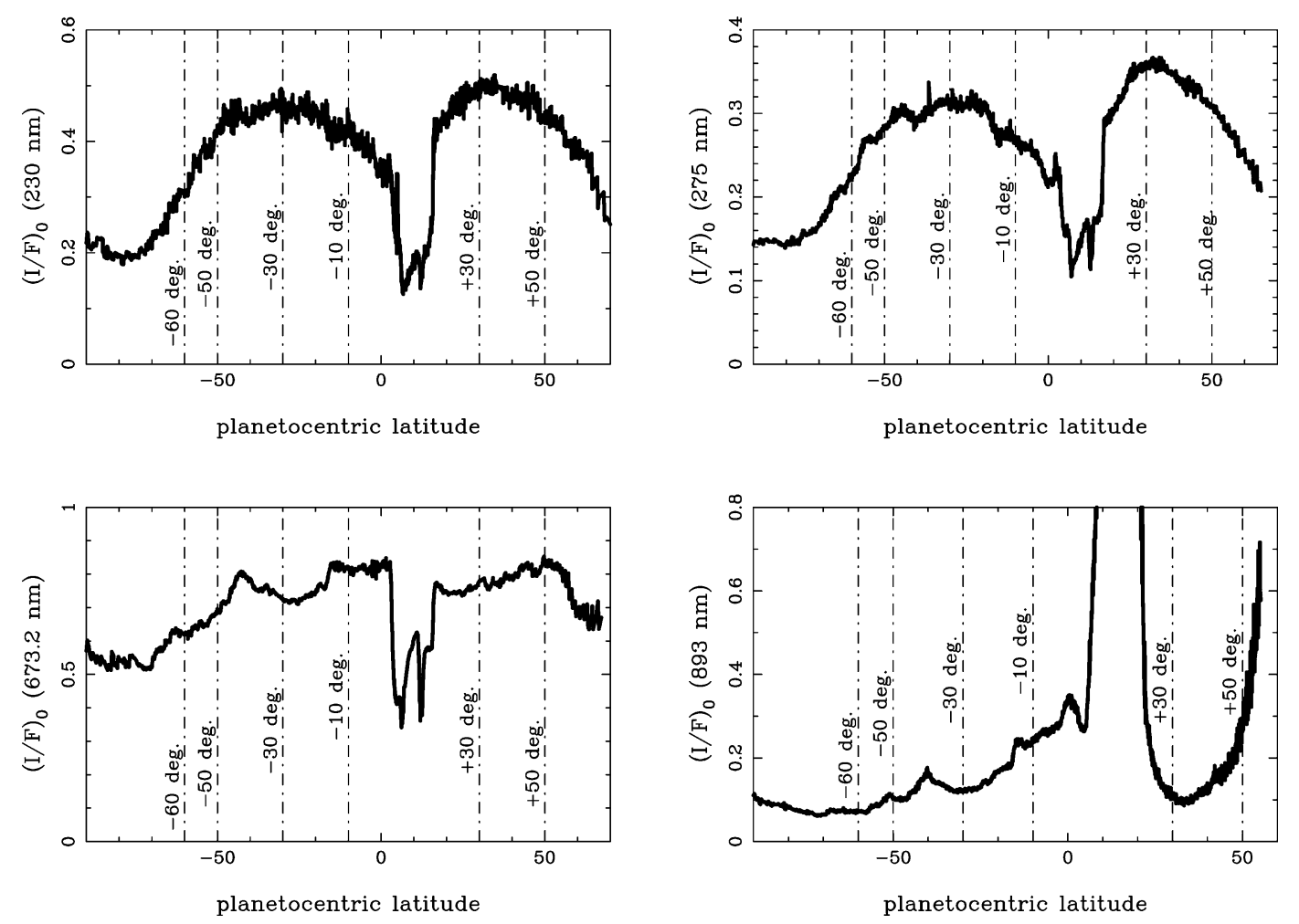

Fig. 2. Geometrically normalized reflectivities at the central meridian.

in Table 1. The image scale was 0.04554 arcsec pixel $^{-1}$, or $281.7 \mathrm{~km}^{-1}$ pixel, at the sub-Earth point at the time of the observations. Saturn's equatorial and polar apparent diameters were 19.38 and 17.37 arcsec, respectively. Overplotted in Fig. 1 we also present a grid of planetocentric latitudes and system III longitudes. The plot shows $15^{\circ}$ spaced parallels and $20^{\circ}$ spaced meridians. The Central Meridian is also overlaid as a vertical line. Saturn displays its structure of belts and zones with absorbing UV aerosols in the south polar cap and the equatorial region. In contrast, the equator appears as a brighter region in the continuum and the methane absorption band.

After calculating the orientation and center of each image, the coordinates were then converted with the above FORTRAN code, to the corresponding cosines of the solar incidence and emission angles $\left(\mu_{0}\right.$ and $\mu$ ), and the azimuthal angle of the ray reflected to the Earth $(\Delta \phi)$.

In Fig. 2 we present the latitudinal behavior of the reflectivity at the four different wavelengths considered in this work $(230,275,673.2$, and $893 \mathrm{~nm})$. In order to correct for geometrical effects we have assumed a Minnaert law $\left(I / F=(I / F)_{0} \mu_{0}^{k} \mu^{k-1}\right)$. The Central Meridian Scans have been divided by a factor $\mu_{0}^{k} \mu^{k-1}$, where $k$ is the Minnaert limb-darkening coefficient. The limb darkening coefficient varies with latitude. However, as mentioned by Tomasko et al. (1984), the latitudinal variations of $k$ on Saturn are significantly smaller than its changes with wavelength. For the purpose of Fig. 2, which is to give a qualitative description of the latitudinal reflectivity behavior, we have assumed $k$ to be constant with latitude. We have obtained the value of $k$ at -10 degrees latitude, at each studied wavelength, giving $k=0.65$ at $230 \mathrm{~nm}, k=0.56$ at $275 \mathrm{~nm}, k=0.9$ at $673.2 \mathrm{~nm}$, and $k=1.3$ at $893 \mathrm{~nm}$. This value has been assumed constant at all latitudes. In Fig. 2 we also indicate the six regions under study in this work. We have obtained limb-to-limb reflectivity scans along the parallels at six different planetocentric latitudes, $-60^{\circ},-50^{\circ},-30^{\circ},-10^{\circ}$, $+30^{\circ}$, and $+50^{\circ}$. We have chosen these latitudes so that the reflectivity scans were not contaminated by the brightness/absorption of the rings or ring shadows. In addition, by comparing the results at both hemispheres we can search for seasonal effects in Saturn's atmosphere. Near the equator, the shadow of the rings is observed at the UV and $673.2 \mathrm{~nm}$. In the methane absorption band the rings region emerges as the brightest region on the planet. To see in more detail the features at the other regions on the disk, we have changed the scale of the plot so that we are not showing the maximum reflectivity value at the rings region. From a mere visual inspection of these plots we can conclude that the equatorial region stands out in the images in the 673.2 and $893 \mathrm{~nm}$ bands. This appearance of the atmosphere of Saturn seems to be long lived, being also detected by other authors at different dates (see, e.g., Owen, 1969, and West et al., 1982). In addition, the southern mid-latitudes are brighter than northern mid-latitudes in the continuum and methane images. 


\section{Retrieval method}

The interpretation of observational data seem to indicate that a simple atmospheric model consisting of a reflecting cloud layer beneath an absorbing gas layer or a vertically homogeneous distribution of scatterers, cannot describe satisfactorily the observations (see, e.g., West et al., 1983a; Tomasko et al., 1984; Acarreta and Sanchez-Lavega, 1999). Acarreta and Sanchez-Lavega (1999) adopted a three-layer model with a gas layer, haze layer and gas and a semiinfinite cloud deck to study the vertical structure of the giant storm erupted in the atmosphere of Saturn in September 1990. Karkoschka and Tomasko $(1992,1993)$ and West et al. (1983a), assumed a cloud structure with a continuous distribution of hazes with aerosols and gas uniformly mixed. However, one of the two structure of clouds proposed by West et al. (1983a), and the structure proposed by Tomasko et al. (1984), include an aerosol free layer between the stratospheric and the upper tropospheric haze. The motivation for this new structure was the impossibility of modeling the polarization measurements of Pioneer 11 by assuming a single layer of scatterers (Danielson and Tomasko, 1969). Stam et al. (2001), have recently retrieved a cloud structure including aerosol free layers between haze layers. By inversion of spectra in the $H(1.45-1.80 \mu \mathrm{m})$ and $K$-band (1.95$2.50 \mu \mathrm{m}$ ) obtained on August 10, 1995, they obtained a cloud structure with two atmospheric regions (stratospheric haze and tropospheric haze) with relatively high scattering densities separated from each other by regions relatively free of scatterers. This atmospheric structure was also successfully used in forward radiative calculations by Ortiz et al. (1996). Taking into account the results obtained by all these authors we have assumed as starting point in our fitting algorithm an atmospheric distribution consisting of three atmospheric layers: an optically thick cloud of ammonia crystals, a tropospheric haze, and a stratospheric haze with aerosol-free layers between clouds and haze layers. In Fig. 3 we show a schematic picture of our initial cloud structure (structure type I). In case we could not find a good fit with this initial cloud structure the pressure levels defining each layer can be changed within the limits of sensitivity of our model so that as a final point of our fitting procedure an atmospheric structure without clear gas layers between haze layers is also allowed (structure type II in Fig. 3).

The radiative transfer discrete-ordinates code DISORT (Stamnes et al., 1988), has been used in combination with a Mie scattering code, which produces a set of optical depths, single scattering albedos and phase functions for multilayer models having a mixture of spherical aerosol particles and gas. Scattering by $\mathrm{He}$ and molecules of $\mathrm{H}_{2}$ is important at short wavelengths. Assuming that the atmosphere is in hydrostatic equilibrium, the Rayleigh optical depth due to these two gases can be expressed as (Tomasko et al., 1984):

$\tau_{R} \simeq 0.0244\left(1+0.016 \lambda^{-2}\right) P \lambda^{-4}$

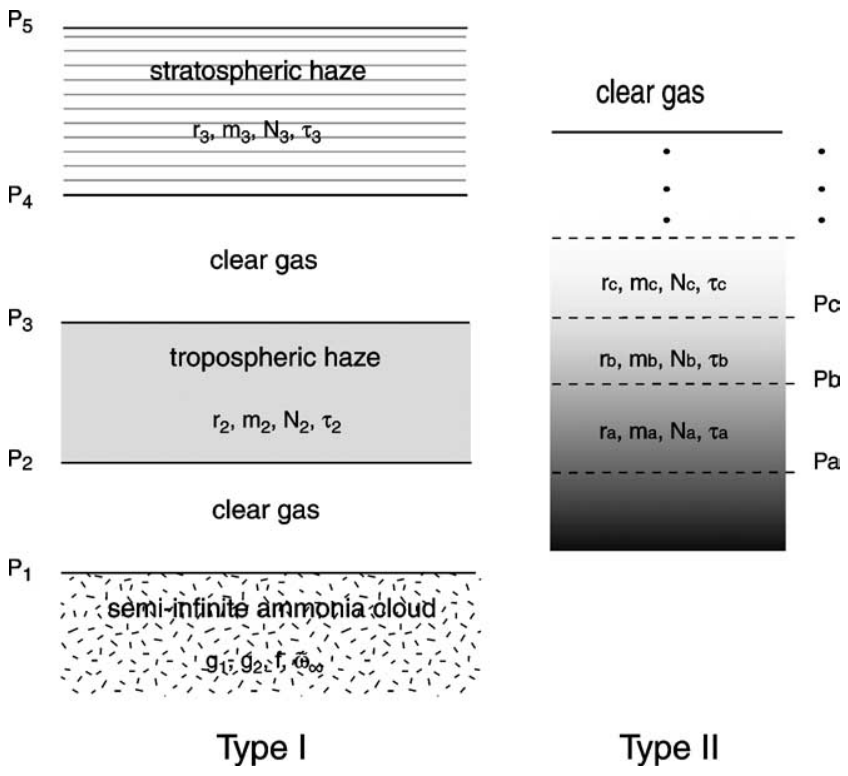

Fig. 3. Schematic diagrams of the proposed type I and type II vertical structure of Saturn's atmosphere.

where $\lambda$ is the wavelength expressed in $\mu \mathrm{m}$, and $P$ is the total pressure expressed in bar.

We adopted the $P(T)$ profile for pressures lower than 1 bar from Lindal et al. (1985), which was derived from radio occultation measurements. Temperatures at atmospheric pressures greater than 1 bar were taken from Stam et al. (2001), based on an extrapolation of the radio occultation temperature profile according to a dry adiabatic. The gravity was varied from $8.8 \mathrm{~m} \mathrm{~s}^{-2}$ at the equator to $12.8 \mathrm{~m} \mathrm{~s}^{-2}$ at the poles, according to values provided by Hubbard and Marley (1989). Volume mixing ratios of $\mathrm{H}_{2}, \mathrm{He}$ and $\mathrm{CH}_{4}$ were fixed to 0.94, 0.058 (Conrath and Gautier, 2000), and 0.002 (Prinn et al., 1984), respectively. The methane absorption coefficients have been calculated by convolution of the coefficients given by Karkoschka (1994), with the appropriate filter transmission curves. Recent measurements of the absorption coefficients of methane has been performed by O'Brien and Cao (2002). The resulting coefficients show good agreement with Karkoschka's coefficients.

To describe the radiative transfer in the atmosphere we have assumed a plane-parallel atmosphere. Since the planeparallel approximation produces erroneous results for small values of the cosines of the solar incidence and emission angles, $\mu$ and $\mu_{0}$ (see, e.g., Van de Hulst, 1980), only pixels with both $\mu>0.15$ and $\mu_{0}>0.15$ are considered for the calculations reported in this paper.

For a given set of initial model parameters we have calculated the reflectivity data for a specific geometry and compared the results with our observations at the same geometry. Subsequently, the values of our free parameters have been changed in order to get the best fit to the limb-to-limb scans at the six latitudes under study. The fitting procedure was carried out in two steps as follows: First we varied all free parameters within certain values discussed below by running 
the model in a nested loop sequence in which the parameters were varied from their minimum to their maximum allowed values. In this way we found the parameter range in which the agreement with the observational reflectivity data was better than $20 \%$. Second, only those parameter combinations leading to reflectivity errors smaller than $20 \%$ were investigated separately by finer parameter grids. From those values we obtained the combination of parameters having an agreement within $10 \%$ of the observational data at each wavelength. This second step restricted the parameter solution space to an optimal combination at each investigated latitude. We consider a fit a good fit when relative errors between calculated and observed absolute reflectivities are smaller than 10 percent for the points defining a limb-to-limb scan according to the photometric uncertainties as described in Section 2. Due to the large amount of free parameters of our model we cannot consider our results as a unique solution. However, we have constrained the values of the input parameters of our model so that they would have physical meaning.

As mentioned the pressure levels defining each layer could be changed within the limits of sensitivity of our model so that as a final point of our fitting procedure an atmospheric structure without clear gas layers between haze layers is also allowed. In each layer we have taken into account absorption and scattering by aerosols and gas. The column density of aerosols in each layer is given by $N$, and the aerosols are distributed in size according to a log-normal size-distribution:

$n(r)=\frac{\exp \left[-0.5\left(\ln (r)-\ln \left(r_{m}\right)\right)^{2} /(\ln (\sigma))^{2}\right]}{\sqrt{2 \pi} r \ln (\sigma)}$,

where $r_{m}$ is the modal radius, $\sigma$ is the width of the distribution. To limit the number of free parameters, we have set $\sigma$ to 1.2. This value makes the size distribution broad enough to avoid resonance effects without making the distribution extremely wide. We have checked the influence of this parameter on our final results. We have changed sigma from 1.1 to 1.4 in steps of 0.1 . The use of a different value of sigma has a slight influence on the final results but do not change the general behavior reported in the paper. For instance, by assuming sigma equal to 1.4 instead of 1.2 the model is slightly darker in the UV and continuum filters and slightly brighter for the methane absorption band. We find the opposite effect by decreasing the value of sigma.

The top boundary of the ammonia cloud was located at 1800 mbar in all the studied cases (Karkoschka and Tomasko, 1992). Since our observations at $673.2 \mathrm{~nm}$ only sound the upper region of the ammonia cloud (the sensitivity limits of the model at the other filters are located at higher levels), we have approximated this cloud by a semi-infinite two-term Henyey-Greenstein function given by:

$$
P\left(g_{1}, g_{2}, f_{1}, \theta\right)=f_{1} P_{\mathrm{HG}}\left(g_{1}, \theta\right)+\left(1-f_{1}\right) P_{\mathrm{HG}}\left(g_{2}, \theta\right)
$$

with

$P_{\mathrm{HG}}(g, \Theta)=\frac{1-g^{2}}{\left(1+g^{2}-2 g \cos \Theta\right)^{3 / 2}}$,

where $\theta$ is the scattering angle, $-1<g<1$ is called the asymmetry parameter that is positive when the scattering predominates in the forward direction, negative if it predominates in the backward direction, and zero for symmetric phase functions, and $0 \leqslant f_{1} \leqslant 1$ is a weighting factor that gives the fraction of radiation scattered into the forward hemisphere. The values for the two-term Henyey-Greenstein parameters and the single scattering albedo, $\left(\varpi_{\infty}\right)$, for the different wavelengths, have been taken from Tomasko and Doose (1984), being $g_{1}=0.633$, $g_{2}=-0.286, f=0.776$, and $\varpi_{\infty}=0.986$. These values were based on polarimetry and photometry data from Pioneer 11 .

For the other atmospheric layers we have considered as free parameters in the model the size of the particles, the pressure levels in which they are distributed, their column abundances, and their complex refractive indices $(m=n+$ $i k)$.

As initial value for the modal radius for tropospheric aerosols, $r_{2}$, we have chosen $1.5 \mu \mathrm{m}$ (Karkoschka and Tomasko, 1993). This initial value has been varied between 1.0 and $2.0 \mu \mathrm{m}$ in steps of $0.2 \mu \mathrm{m}$. In addition, Polarization observations at 0.44 and $0.64 \mu \mathrm{m}$ from Pioneer 11 (Tomasko and Doose, 1984) seem to indicate the existence of rather small aerosols (radii between 0.1 and $0.2 \mu \mathrm{m}$ ) at stratospheric levels. Therefore, we have varied the modal radius, $r_{3}$, of the aerosols in the stratospheric haze between those values in steps of $0.02 \mu \mathrm{m}$. The values of $p_{2}, p_{3}$, $p_{4}$, and $p_{5}$ were varied along the most extreme values obtained by Ortiz et al. (1996), and Stam et al. (2001), i.e., $100 \mathrm{mbar} \leqslant p_{2} \leqslant 1600 \mathrm{mbar} ; 60 \mathrm{mbar} \leqslant p_{3} \leqslant 170 \mathrm{mbar}$; 20 mbar $\leqslant p_{4} \leqslant 60$ mbar; 10 mbar $\leqslant p_{5} \leqslant 30$ mbar. In order to minimize the number of free parameters in the model, the real part of the refractive index $n$ was fixed to 1.43 , which corresponds to the value for the expected condensates in Saturn's stratosphere: $\mathrm{C}_{4} \mathrm{H}_{2}, \mathrm{C}_{2} \mathrm{H}_{6}, \mathrm{P}_{2} \mathrm{H}_{4}, \mathrm{NH}_{3}$ (Karkoschka and Tomasko, 1993). Nevertheless, we also checked the sensitivity of the model to this fixed parameter, not finding any significant differences in the results after varying $n$ between 1.4 and 1.55. As a starting point for the imaginary part of the refractive indices we have taken the results obtained by Karkoschka and Tomasko (1993). From these initial values we let $k$ vary until a good fit with the observed reflectivity data was obtained. In Table 2 we summarize the initial assumptions for the free parameters of our model.

We note that in our model, as a first approximation, we are considering spherical particles although, as pointed out by (Tomasko et al., 1984), particles in Saturn atmosphere are expected to be irregular. However, there is not a numerical code that can reproduce the single scattering properties for the broad range of particle shapes and sizes expected to find in the atmosphere of Saturn. Therefore, it is difficult to 
Table 2

Initial assumptions for the free parameters of the model: $r_{2}$ and $r_{3}$ are the modal radius for tropospheric and stratospheric aerosols respectively; $p_{2}$ and $p_{3}$ are the pressures at, respectively the bottom and top of the tropospheric haze, whereas $p_{4}$ and $p_{5}$ are the pressure at the bottom and top of the stratospheric haze respectively; $k_{2}$ and $k_{3}$ are the values of the imaginary part of the refractive index for the tropospheric and stratospheric aerosols at the different wavelengths studied in this work

\begin{tabular}{lcc}
\hline Parameter & Studied range & Reference \\
\hline$r_{2}(\mu \mathrm{m})$ & $1.0 \leqslant r \leqslant 2.0$ & Karkoschka and Tomasko (1993) \\
$r_{3}(\mu \mathrm{m})$ & $0.1 \leqslant r \leqslant 0.2$ & Tomasko and Doose (1984) \\
$p_{2}(\mathrm{mbar})$ & $100 \leqslant p \leqslant 1600$ & Ortiz et al. (1996); Stam et al. (2001) \\
$p_{3}(\mathrm{mbar})$ & $60 \leqslant p \leqslant 170$ & Ortiz et al. (1996); Stam et al. (2001) \\
$p_{4}(\mathrm{mbar})$ & $20 \leqslant p \leqslant 60$ & Ortiz et al. (1996); Stam et al. (2001) \\
$p_{5}(\mathrm{mbar})$ & $10 \leqslant p \leqslant 30$ & Ortiz et al. (1996); Stam et al. (2001) \\
$k_{2}(\mathrm{UV})$ & $6.5 \times 10^{-3}$ & Karkoschka and Tomasko (1993) \\
$k_{2}(673.2 \mathrm{~nm})$ & $2.0 \times 10^{-4}$ & Karkoschka and Tomasko (1993) \\
$k_{2}(893 \mathrm{~nm})$ & $2.0 \times 10^{-4}$ & Karkoschka and Tomasko (1993) \\
$k_{3}(\mathrm{UV})$ & 0.14 & Karkoschka and Tomasko (1993) \\
$k_{3}(673.2 \mathrm{~nm})$ & $1.0 \times 10^{-4}$ & Karkoschka and Tomasko (1993) \\
$k_{3}(893 \mathrm{~nm})$ & $1.0 \times 10^{-4}$ & Karkoschka and Tomasko (1993) \\
\hline
\end{tabular}

We also show the initial values in the fitting procedure taken from Karkoschka and Tomasko (1993). From these initial values we let $k$ vary to smaller and larger values than the initial one until a good fit with the observational values was obtained.

directly relate aerosol optical properties derived from modeling to constraints on physical properties such as size and refractive indices. Thus, we do not consider the retrieved values for the physical properties of the aerosols as the actual values of particles in the atmosphere, but as a combination of parameters that can reproduce our observational data. These results are of main importance to study the relative differences in the vertical structure of clouds at different latitudes, providing information on the possible relation with seasonal changes, which is the main purpose of this paper.

\section{Results}

In this section we present first the results at the equator and mid-latitudes. The results at the south polar region are given next.

\subsection{Equator and mid-latitudes}

Figures 4-8, show the best fits for the different latitudes at the equator and mid-latitudes studied in this work. The best fits are presented along with the observational data. The values of the free parameters which best fit the observational data at all latitudes are presented in Tables 3-5. From the results of the model we have found an atmospheric structure consisting of two hazes (stratospheric and tropospheric) separated by clear gas layers.

Once the best fit at a certain latitude was found we checked the sensitivity of the best model solution to a single free parameter of the model. As examples, in Figs. 4-8, we also present the calculated reflectivity data by varying different free parameters around the values obtained for the best
Table 3

Calculated pressure levels and modal radius at the six studied latitudes, $p_{2}$ and $p_{3}$ are the pressures at the bottom and the top of the tropospheric haze respectively; and $p_{4}$ and $p_{5}$ are the pressure at the bottom and top of the stratospheric haze, respectively

\begin{tabular}{lcccccc}
\hline Latitude & $p_{2}(\mathrm{mbar})$ & $r_{2}(\mu \mathrm{m})$ & $p_{3}(\mathrm{mbar})$ & $p_{4}(\mathrm{mbar})$ & $r_{3}(\mu \mathrm{m})$ & $p_{5}(\mathrm{mbar})$ \\
\hline$-50^{\circ}$ & 400 & 1.5 & 87 & 12 & 0.1 & 1 \\
$-30^{\circ}$ & 400 & 1.2 & 60 & 10 & 0.1 & 1 \\
$-10^{\circ}$ & 180 & 1.2 & 60 & 20 & 0.12 & 2 \\
$+30^{\circ}$ & 600 & 1.2 & 90 & 10 & 0.12 & 1 \\
$+50^{\circ}$ & 350 & 1.2 & 140 & 30 & 0.12 & 6 \\
\hline
\end{tabular}

Table 4

Retrieved refractive indices $(m=n+i k)$ at different wavelengths

\begin{tabular}{|c|c|c|c|c|c|c|c|c|}
\hline \multirow[t]{2}{*}{ Latitude } & \multicolumn{2}{|c|}{$230 \mathrm{~nm}$} & \multicolumn{2}{|c|}{$275 \mathrm{~nm}$} & \multicolumn{2}{|c|}{$673.2 \mathrm{~nm}$} & \multicolumn{2}{|c|}{$893 \mathrm{~nm}$} \\
\hline & $k_{2}$ & $k_{3}$ & $k_{2}$ & $k_{3}$ & $k_{2}$ & $k_{3}$ & $k_{2}$ & $k_{3}$ \\
\hline$-50^{\circ}$ & 0.0045 & 0.001 & 0.0045 & 0.001 & 0.0002 & 0.0001 & 0.0001 & .00 \\
\hline$-30^{\circ}$ & 0.0015 & 0.001 & 0.0025 & 0.001 & 0.0002 & 0.0 & 0.0001 & 0.00 \\
\hline$-10^{\circ}$ & 0.001 & 0.001 & 0.0025 & 0.001 & 0.0001 & 0.0001 & 0.0001 & 0.00 \\
\hline$+30^{\circ}$ & 0.0025 & 0.001 & 0.002 & 0.001 & 0.0002 & 0.001 & 0.0001 & 0.00 \\
\hline$+50^{\circ}$ & 0.006 & 0.009 & 0.0035 & 0.003 & 0.0002 & 0.0001 & 0.0001 & 0.000 \\
\hline
\end{tabular}

The real part $n$ was fixed to 1.43 in all cases, $k_{2}$ corresponds to the tropospheric haze, and $k_{3}$ corresponds to the stratospheric haze.

Table 5

Calculated optical depths at the six different latitudes and filters studied in this work

\begin{tabular}{|c|c|c|c|c|c|c|c|c|}
\hline \multirow[t]{2}{*}{ Latitude } & \multicolumn{2}{|c|}{$230 \mathrm{~nm}$} & \multicolumn{2}{|c|}{$275 \mathrm{~nm}$} & \multicolumn{2}{|c|}{$673.2 \mathrm{~nm}$} & \multicolumn{2}{|c|}{$893 \mathrm{~nm}$} \\
\hline & $\tau_{2}$ & $\tau_{3}$ & $\tau_{2}$ & $\tau_{3}$ & $\tau_{2}$ & $\tau_{3}$ & $\tau_{2}$ & $\tau_{3}$ \\
\hline$-50^{\circ}$ & 27.1 & 0.29 & 25.5 & 0.18 & 25.9 & 0.013 & 28.0 & 0.09 \\
\hline$-30^{\circ}$ & 19.1 & 0.27 & 17.1 & 0.17 & 17.2 & 0.013 & 17.8 & 0.08 \\
\hline$-10^{\circ}$ & 33.0 & 0.51 & 32.4 & 0.34 & 35.3 & 0.033 & 32.9 & 0.17 \\
\hline$+30^{\circ}$ & 21.0 & 0.41 & 18.0 & 0.29 & 17.2 & 0.03 & 16.0 & 0.05 \\
\hline$+50^{\circ}$ & 34.0 & 0.58 & 32.9 & 0.37 & 35.5 & 0.03 & 33.2 & 0.15 \\
\hline
\end{tabular}

$\tau_{2}$ corresponds to the tropospheric haze and $\tau_{3}$ corresponds to the stratospheric haze (see Fig. 3).

fit. Thus, in Fig. 4 we illustrate the sensitivity of our model to the value of $p_{3}$ (the upper tropospheric haze boundary). The sensitivity of the model to the value of the lower boundary of the tropospheric haze, $p_{2}$, is presented in Fig. 5. As shown in Figs. 4 and 5, the pressure levels of the tropospheric haze are best constrained by the observations at $893 \mathrm{~nm}$. A similar test is presented in Fig. 6, in this case we present the sensitivity of the model to the value of the lower boundary of the stratospheric haze, $p_{4}$. We have also found the strongest differences for the methane absorption band. As shown in this figure, the calculated reflectivities as function of the longitude at $893 \mathrm{~nm}$ are very low when comparing with the observational data if we consider $p_{4}$ equal to 2 mbar. The values of the calculated curve increase by increasing the value of $p_{4}$ until 10 mbar. At this point, the calculated reflectivities match the observational data. In contrast, if we continue increasing the value of $p_{4}$ until $40 \mathrm{mbar}$, the calculated curve is again lower than the observational data. In Fig. 7 we have studied the sensitivity of the model to the modal radius, $r_{2}$, of the aerosol particles in the tropospheric 

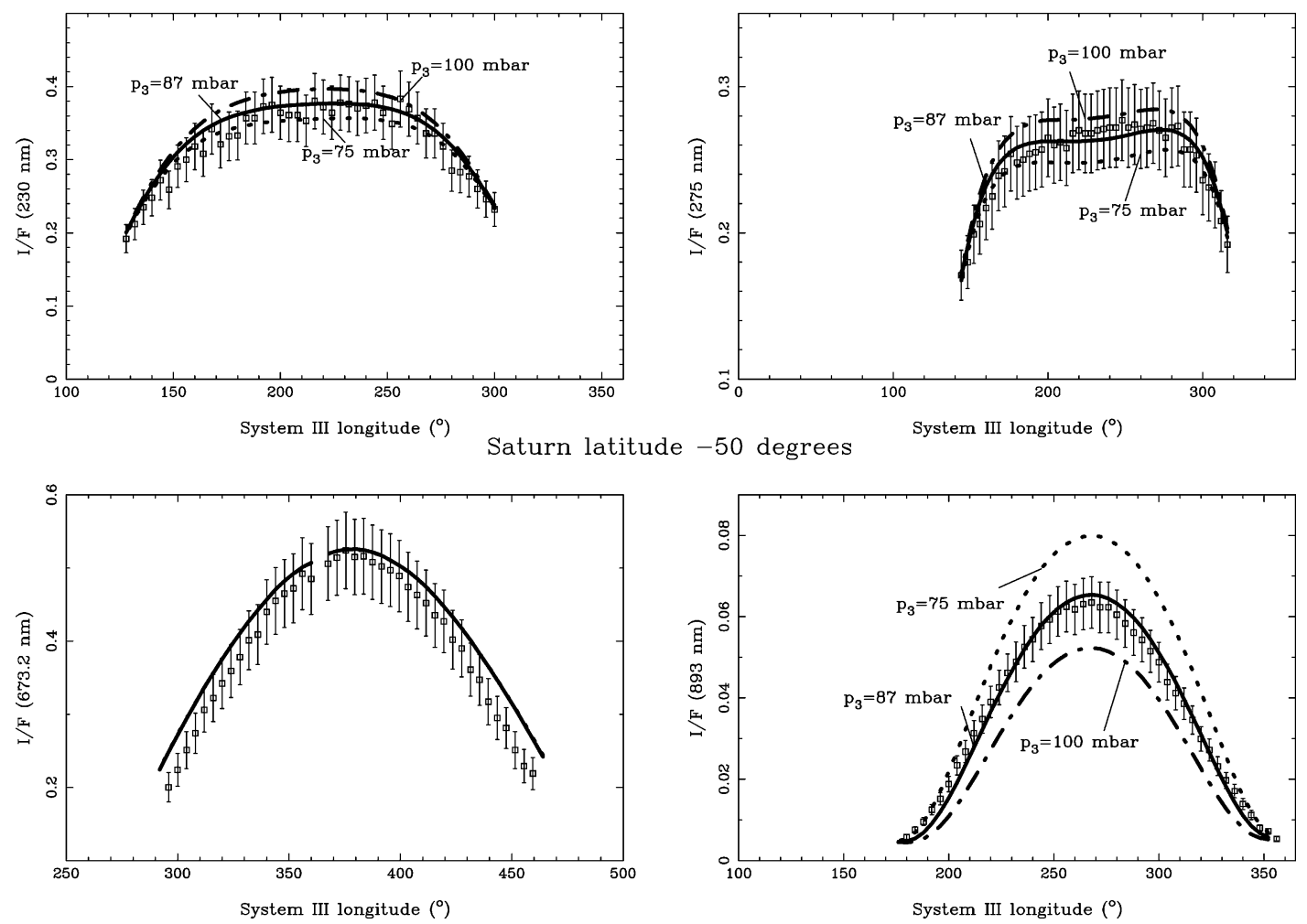

Fig. 4. Observed reflectivities (squares) and best fits of the model (solid lines) at -50 degrees. Note that we have allowed the longitude to be greater than 360 and plot for easy reading of the bottom left plot. For longitudes larger than 360 deg the actual values should be decreased by 360 . We also show the results of the model by varying the values of $p_{3}$ around the best fit values (dotted and dotted-dashed lines). The calculated reflectivity at $673 \mathrm{~nm}$ is not sensitive to the varied parameter. The results for the three different values of $p_{3}$ are on top of each other.
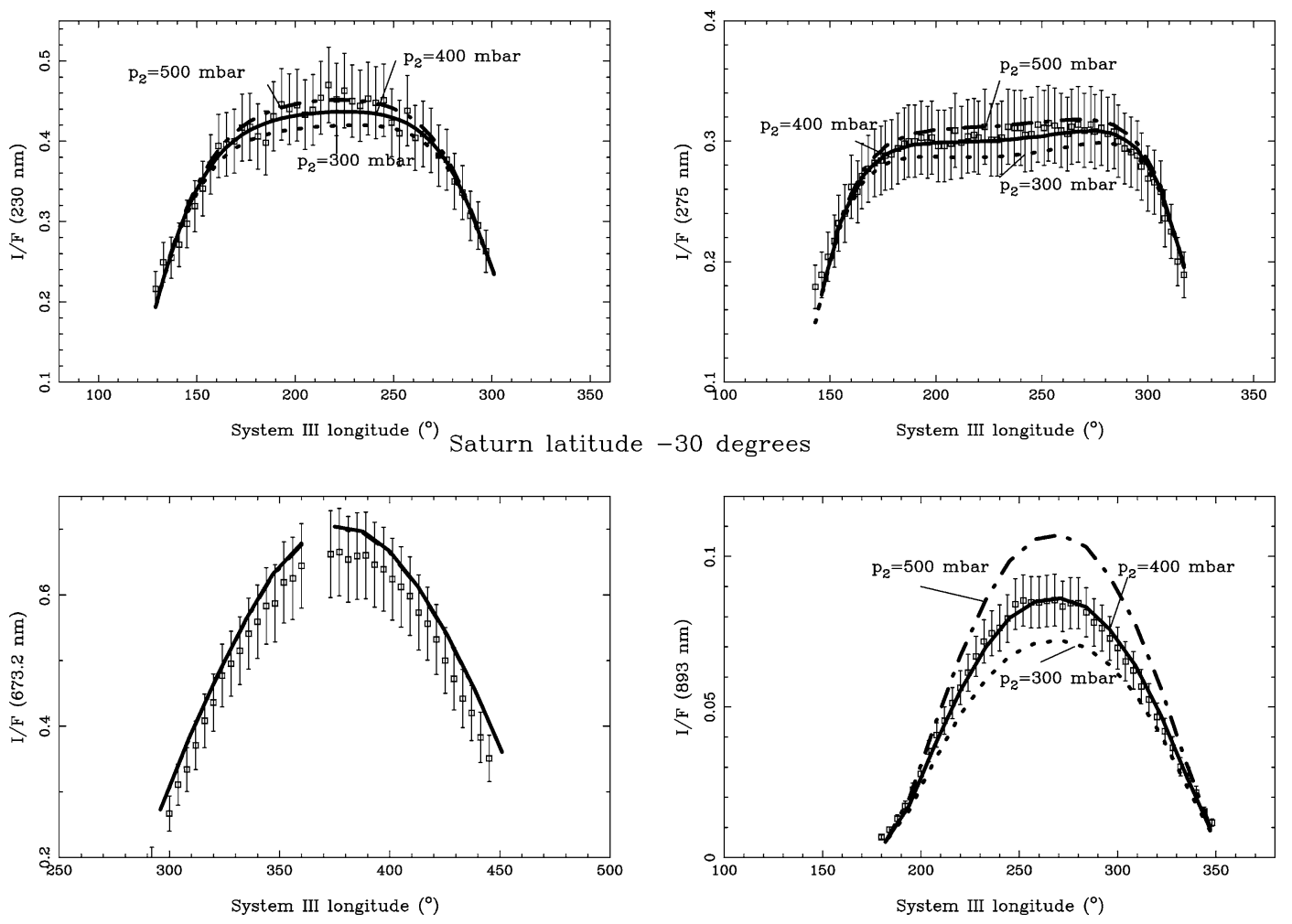

Fig. 5. Same as Fig. 4 but at -30 degrees. In this case we have varied the values of $p_{2}$ around the best fit values (dotted and dotted-dashed lines). The calculated reflectivity at $673 \mathrm{~nm}$ is not sensitive to the varied parameter. The results for the three different values of $p_{2}$ are on top of each other. 

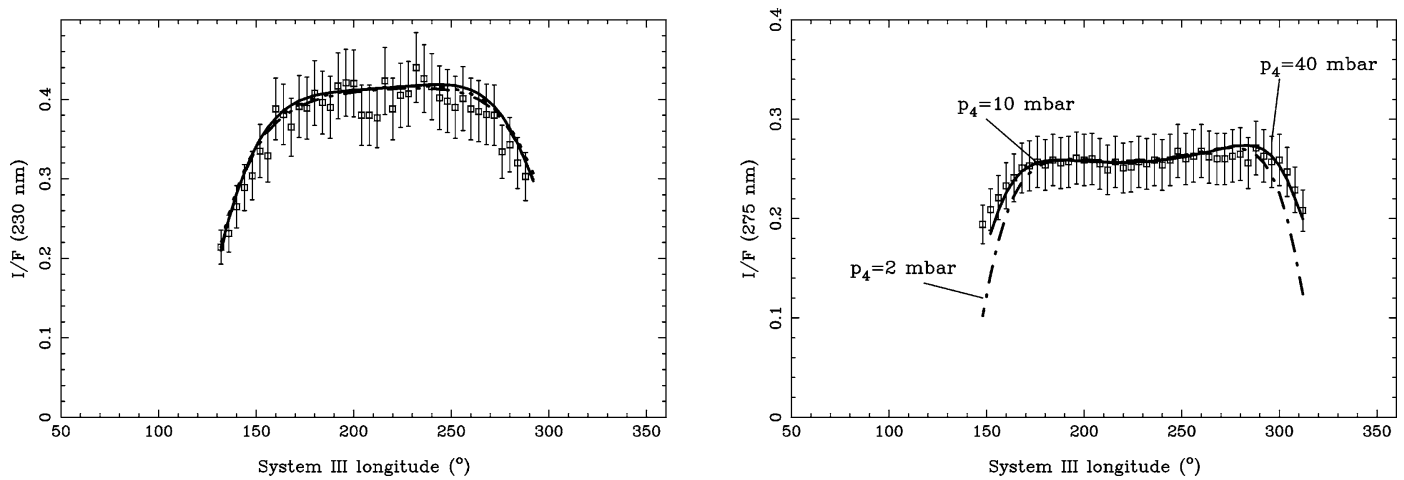

Saturn latitude -10 degrees
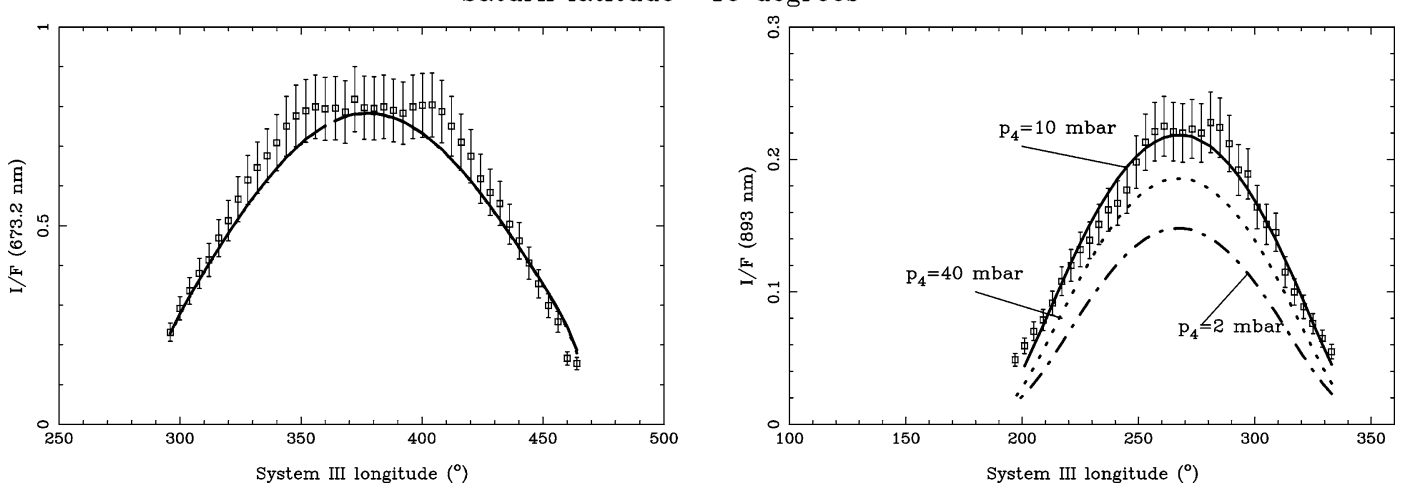

Fig. 6. Same as Fig. 4 but at -10 degrees. In this case we have changed the values of $p_{4}$ around the best fit values (dotted and dotted-dashed lines). The calculated reflectivity at $673 \mathrm{~nm}$ is not sensitive to the varied parameter. The results for the three different values of $p_{4}$ are on top of each other.
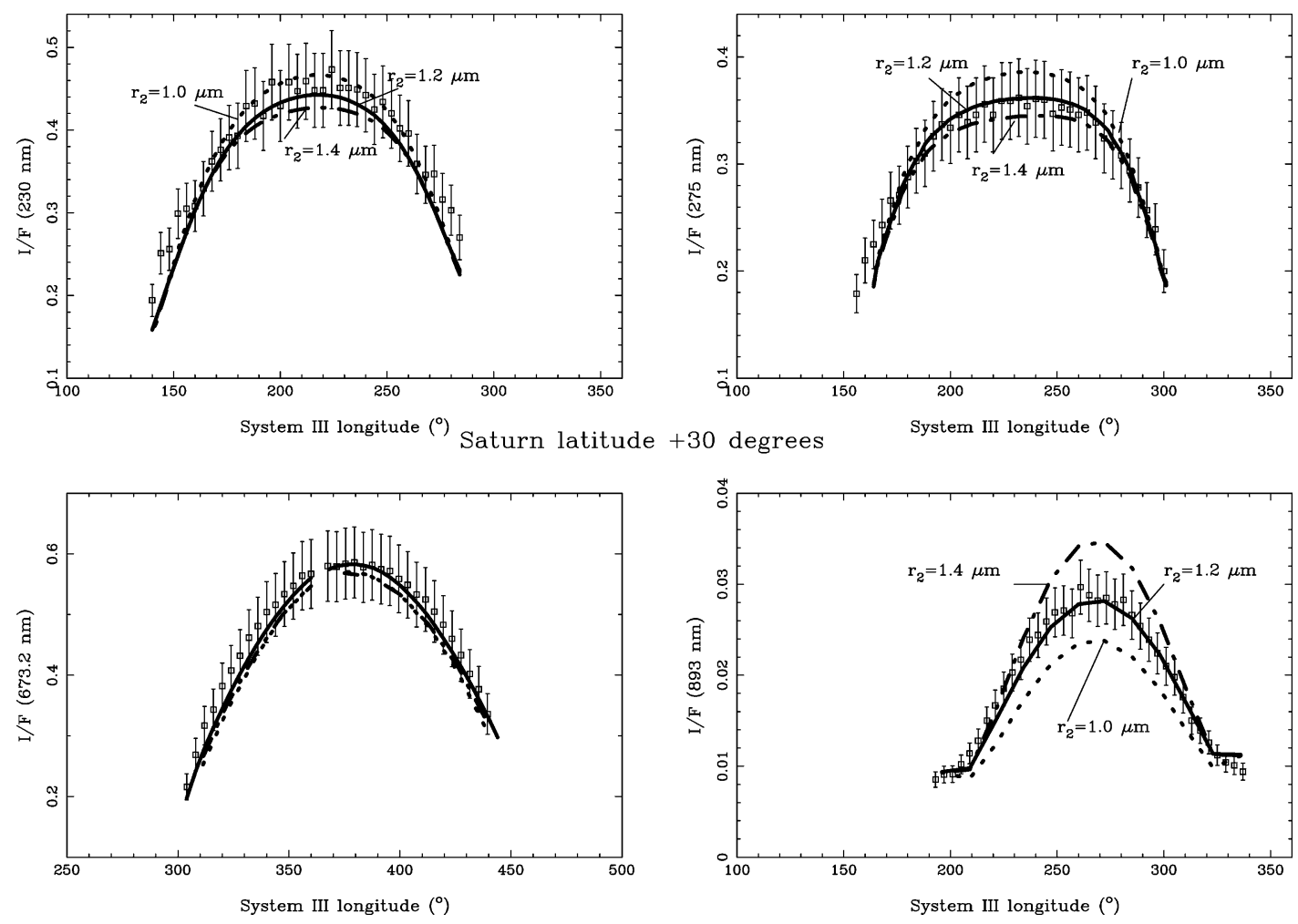

Fig. 7. Same as Fig. 4 but at +30 degrees. We present also the results of the model varying $r_{2}$ around the best fit values (dotted and dotted-dashed lines). 

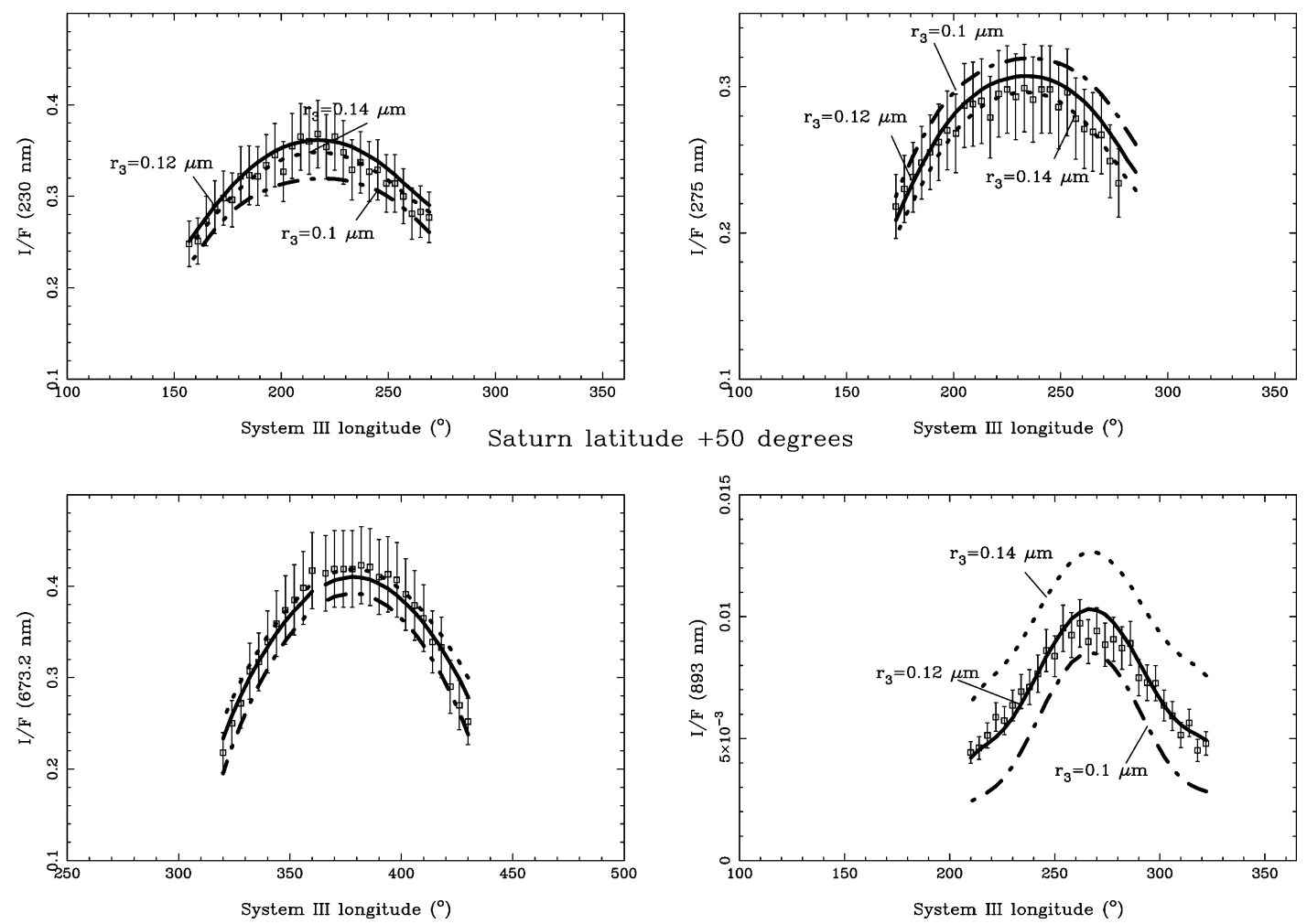

Fig. 8. Same as Fig. 4 but at +50 degrees. We present also the results of the model varying $r_{3}$ around the best fit values $(0.14 \mu \mathrm{m}$ dotted and $0.1 \mu \mathrm{m}$ dotted-dashed lines).

haze. Again, the wavelength at which the modeled reflectivities show the largest sensitivity appears to be that of the methane absorption band ( $893 \mathrm{~nm}$ ), although all the other filters show marked deviations from the best fitted curve when altering the best-fitted $r_{2}$. We have also studied the sensitivity of the model to the values of $r_{3}$. The results are presented in Fig. 8 together with the best fitted values. As can be seen, changes in $r_{3}$ affect all wavelengths, with the most dramatic effects at the far UV and $893 \mathrm{~nm}$ wavelengths.

\subsection{Latitude $-60^{\circ}$}

As mentioned in Section 3, as a starting point in the fitting procedure, we have assumed an atmospheric distribution consisting of three atmospheric layers in which the physical parameters of the clouds (pressure levels, column density, size, and refractive indices of the aerosols) have been iteratively changed until a good match between observational data and calculated values is obtained. Such atmospheric structure has produced satisfactory results at all latitudes studied in this work except at $-60^{\circ}$. In this case we needed a multilayered structure with particles located between 400 mbar and 0.4 mbar without clear gas layers between different haze layers (see Fig. 13). Even so, there is still some discrepancy with the observational data points near the limb at $673 \mathrm{~nm}$. At present, we cannot explain the origin of this discrepancy. In Table 6 , we present the calculated atmospheric model at $-60^{\circ}$. In Fig. 9 the best fitted values are presented together with the calculated reflectivity data by varying $p_{g 2}$ (the upper boundary of the cloud structure) around the values obtained for the best fit.

In order to show the need of a different cloud structure at -60 degrees, in Fig. 10 we present the calculated reflectivity data for the multilayer structure described in Table 6 together with the best fit obtained for a cloud structure type I. In this case, the tropospheric cloud had to be located from 130 to 600 mbar with particles of $1.5 \mu \mathrm{m}$ in modal radius. The stratospheric haze was situated between 10 and 30 mbar with particles of $0.15 \mu \mathrm{m}$ in modal radius. The imaginary part of the refractive index for particles at the stratospheric and tropospheric cloud was fixed to 0.07 at $230 \mathrm{~nm}$ and 0.05 at $275 \mathrm{~nm}$, being 0.0001 for both clouds at 673 and $893 \mathrm{~nm}$. As shown in that figure we could obtain a reasonable good fit for the continuum and methane absorption band filters. However, with such cloud distribution we could not properly fit the observational data for the UV filters, specially the data at $230 \mathrm{~nm}$.

After obtaining the cloud structure at the south polar region we checked whether a vertical structure such as the one obtained at -60 degrees would fit the observational data at other latitudes on the planet. Thus, in our fitting procedure we assumed as a starting point the cloud structure obtained at -60 degrees letting all parameters to change within the ranges discussed in Section 3. In Fig. 11 we present the results for the best fit obtained at -50 degrees considering a multilayered structure with particles located between 1 and 400 mbar. The $r_{m}$ of the particles varies among 
Table 6

Calculated physical parameters of the cloud structure at latitude $-60^{\circ}$

\begin{tabular}{|c|c|c|c|c|c|c|c|c|c|c|c|}
\hline \multirow[t]{2}{*}{ Layer } & \multirow[t]{2}{*}{$p_{\text {lay1 }}$ (mbar) } & \multirow[t]{2}{*}{$p_{\text {lay2 }}$ (mbar) } & \multirow[t]{2}{*}{$r_{\text {lay }} \mu \mathrm{m}$} & \multicolumn{2}{|c|}{$230 \mathrm{~nm}$} & \multicolumn{2}{|c|}{$275 \mathrm{~nm}$} & \multicolumn{2}{|c|}{$673.2 \mathrm{~nm}$} & \multicolumn{2}{|c|}{$893 \mathrm{~nm}$} \\
\hline & & & & $k_{\text {lay }}$ & $\tau_{\text {lay }}$ & $k_{\text {lay }}$ & $\tau_{\text {lay }}$ & $k_{\text {lay }}$ & $\tau_{\text {lay }}$ & $k_{\text {lay }}$ & $\tau_{\text {lay }}$ \\
\hline $\mathrm{g}$ & 17 & 0.04 & 0.07 & 0.09 & 0.31 & 0.07 & 0.01 & 0.0001 & 0.009 & 0.0001 & 0.16 \\
\hline f & 28 & 17 & 0.08 & 0.09 & 0.32 & 0.07 & 0.20 & 0.0001 & 0.019 & 0.0001 & 0.06 \\
\hline $\mathrm{e}$ & 48 & 28 & 0.08 & 0.02 & 0.43 & 0.02 & 0.25 & 0.0001 & 0.026 & 0.0001 & 0.1 \\
\hline d & 82 & 48 & 0.10 & 0.02 & 1.74 & 0.02 & 1.2 & 0.001 & 0.21 & 0.001 & 0.23 \\
\hline $\mathrm{c}$ & 141 & 82 & 0.10 & 0.009 & 3.12 & 0.009 & 2.18 & 0.001 & 0.31 & 0.001 & 0.39 \\
\hline b & 243 & 141 & 0.20 & 0.009 & 8.0 & 0.009 & 8.2 & 0.001 & 4.95 & 0.001 & 3.37 \\
\hline $\mathrm{a}$ & 400 & 243 & 1.0 & 0.009 & 147.6 & 0.009 & 148.6 & 0.001 & 167.8 & 0.001 & 155.9 \\
\hline
\end{tabular}

$p_{\text {lay } 1}$ and $p_{\text {lay } 2}$ correspond to the calculated maximum and minimum pressure levels that determine a layer according to Fig. 13, where the suffix "lay" may run from " $a$ " to " $g$ " according to the layer nomenclature; $r_{\text {lay }}, k_{\text {lay }}$, and $\tau_{\text {lay }}$ correspond to the modal radius, imaginary part of the refractive index, and optical depths of each layer (see Fig. 13). The real part of the refractive index $n$ was fixed to 1.4 at $230 \mathrm{~nm}$ and $275 \mathrm{~nm}$, and to 1.6 at $673.2 \mathrm{~nm}$ and $893 \mathrm{~nm}$ in all layers.
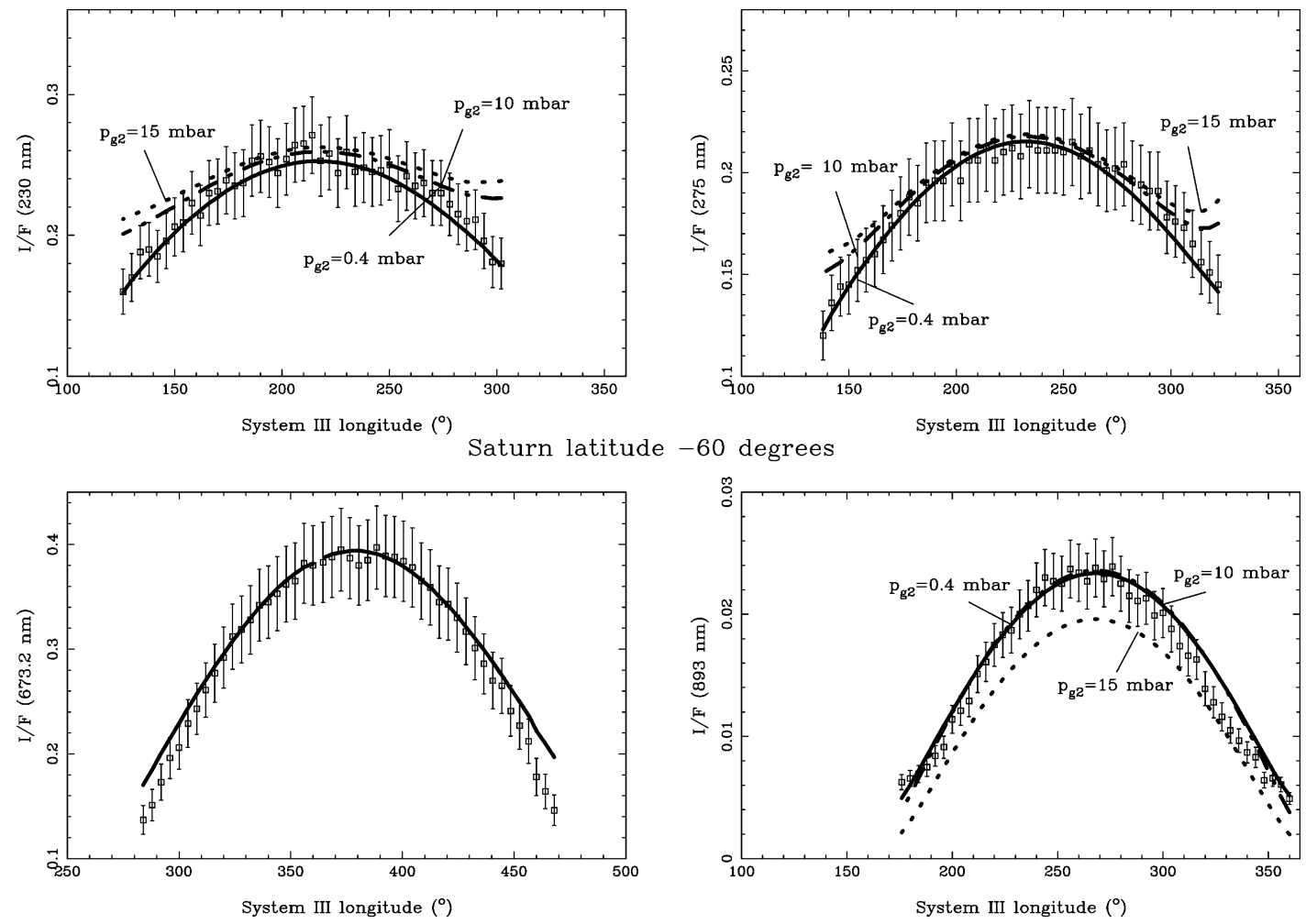

Fig. 9. Same as Fig. 4 but at -60 degrees. We also show the results of the model by varying the values of $p_{g 2}$ around the best fit values (dotted and dotted-dashed lines). The calculated reflectivity at $673 \mathrm{~nm}$ is not sensitive to the varied parameter. The results for the three different values of $p_{g 2}$ are on top of each other.

0.07 and $0.2 \mu \mathrm{m}$ for pressure levels from 1 to $300 \mathrm{mbar}$ with particles of $1 \mu \mathrm{m}$ in modal radius between 300 and 400 mbar. As shown in Fig. 11, the fit to the observational data points near the limb at $673 \mathrm{~nm}$ is slightly better than that obtained with the model having the type I structure (atmospheric structure consisting of a stratospheric haze and a tropospheric haze interspersed by clear gas regions). However, we could not find simultaneously a reasonable good fit at $893 \mathrm{~nm}$.

Similar results were obtained at -30 degrees (Fig. 12). Again, the fit at $673 \mathrm{~nm}$ could be improved with respect to the cloud structure type I. At the same time the fits for the UV filters assuming the same cloud distribution are quite good. However, we could not fit the observational data at $893 \mathrm{~nm}$ with the type II structure models.

\section{Discussion and summary}

Figure 13 shows schematically the retrieved vertical structure of particles at all latitudes studied in this work. According to the retrieved results we can distinguish two different cloud structures on the planet. While at the equator and mid-latitudes the atmospheric structure consists of two hazes (stratospheric and tropospheric) separated by clear gas, the south polar region $\left(-60^{\circ}\right)$ appears as a multilayered 

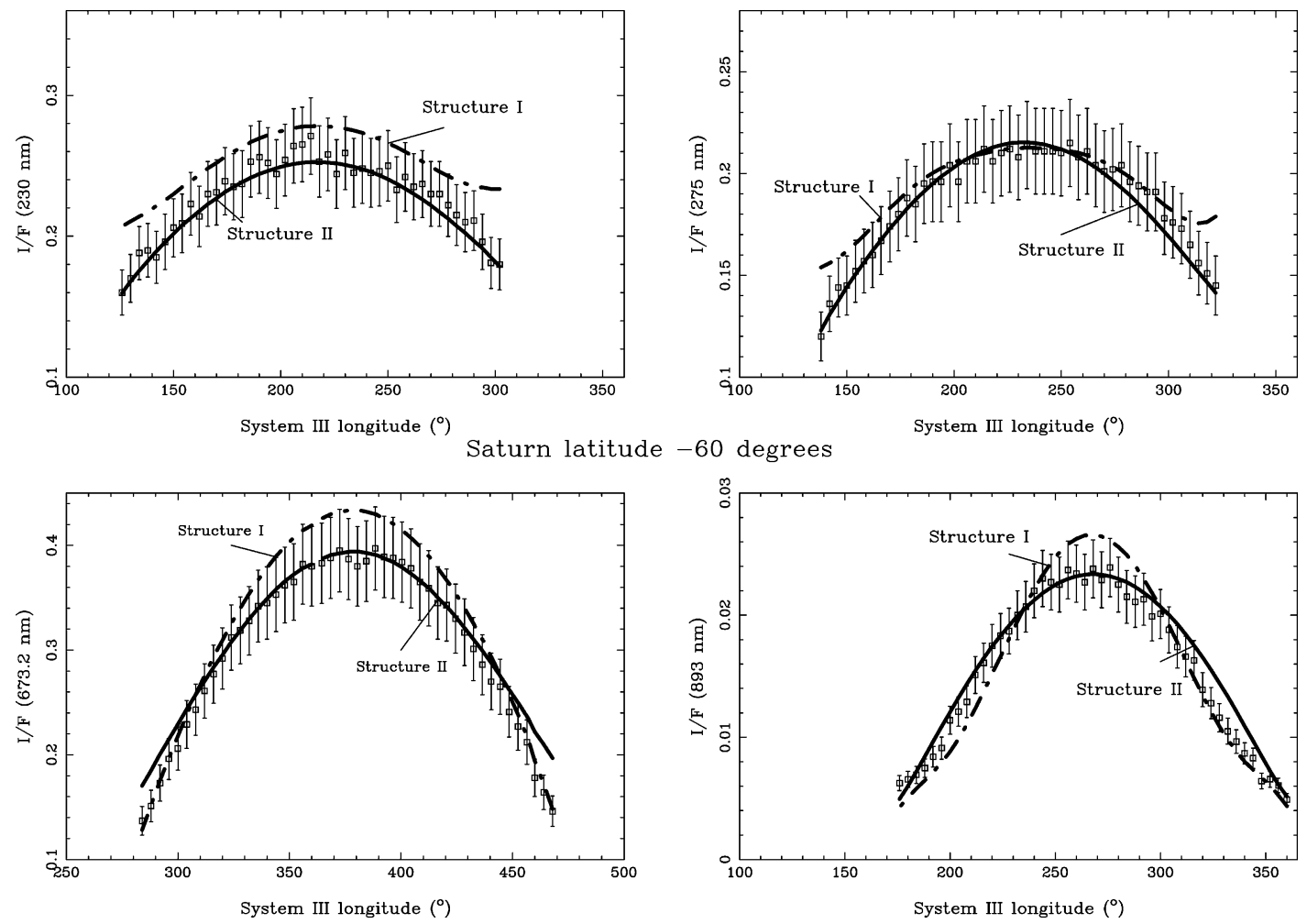

Fig. 10. Observed reflectivities (squares) and best fits of the model for type I structure (dotted-dashed lines) and type II structure (solid lines) at -60 degrees. The observational data are presented together with their error bars.
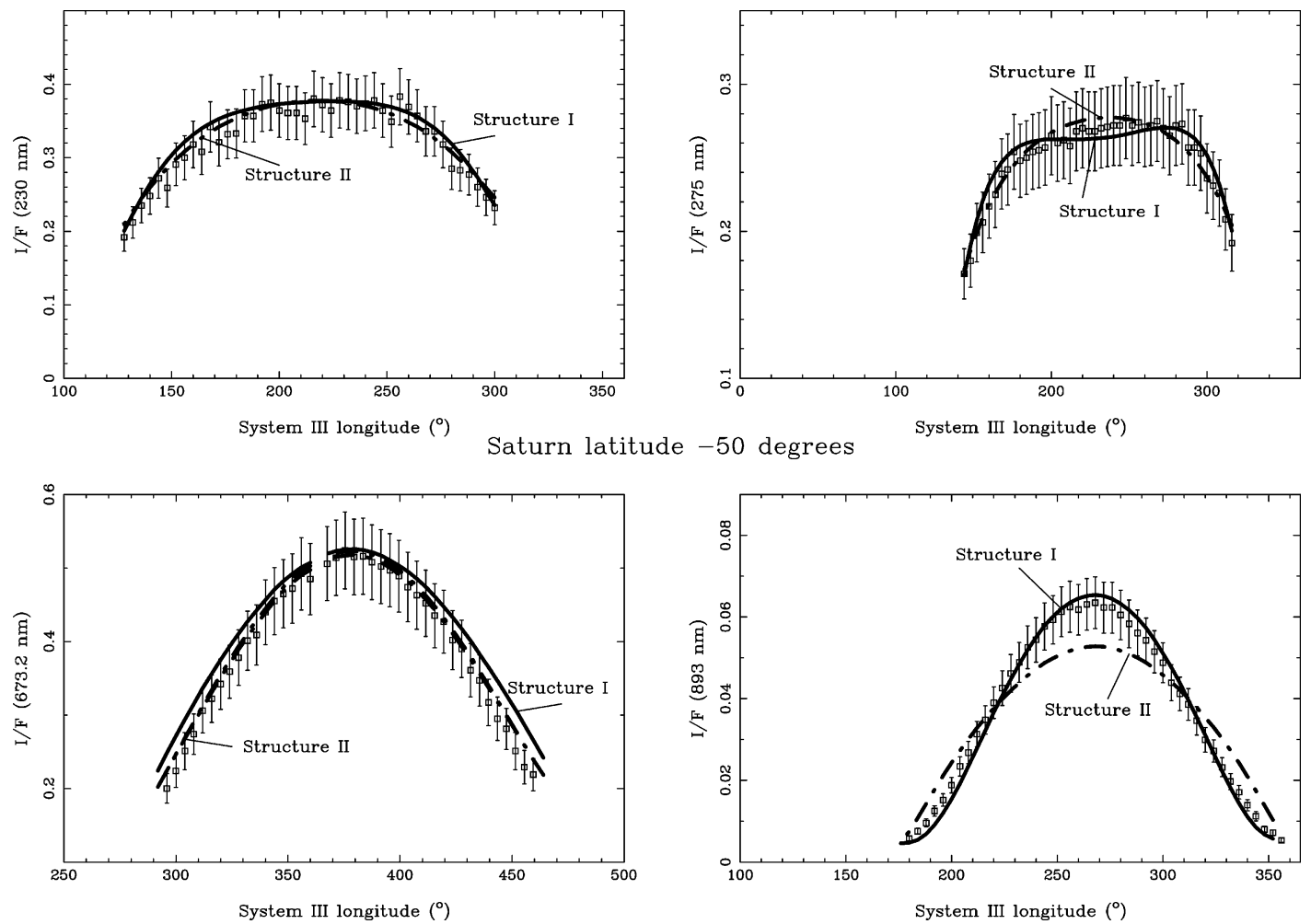

Fig. 11. Same as Fig. 10 but at -50 degrees. 

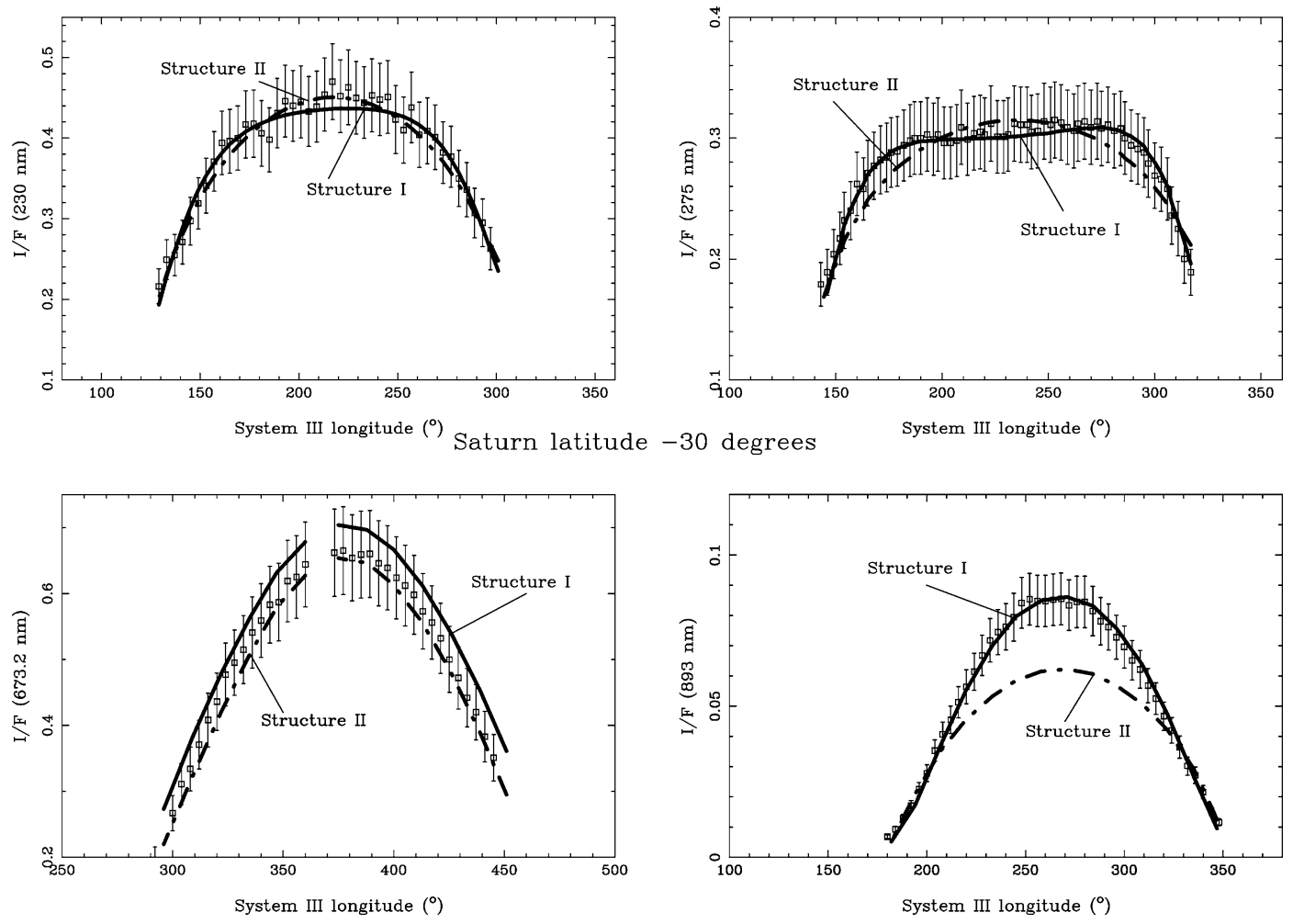

Fig. 12. Same as Fig. 10 but at -30 degrees.

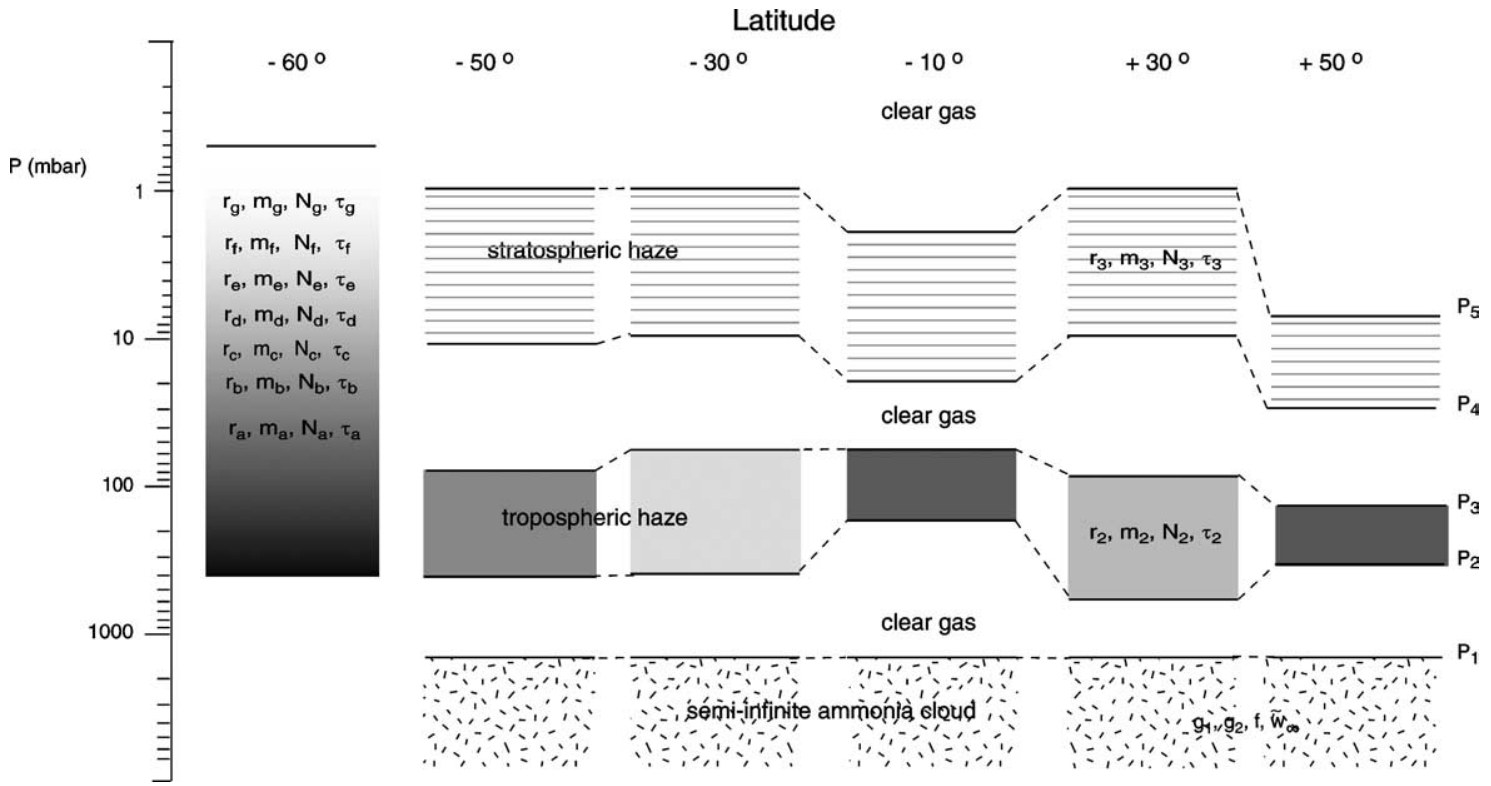

Fig. 13. Retrieved vertical structure of clouds of Saturn's atmosphere at six different latitudes: $-60^{\circ},-50^{\circ},-30^{\circ},-10^{\circ},+30^{\circ}$, and $+50^{\circ}$. On the left column we show the pressure in a logarithmic scale expressed in mbar, with $p_{1}$ the pressure at the top of the semi-infinite ammonia cloud; $p_{2}$ and $p_{3}$ define the location of the tropospheric haze, and $p_{4}$ and $p_{5}$ are the pressure at the bottom and top of the stratospheric haze respectively. At $-60^{\circ}$ we have used a different nomenclature, the different layers of the multilayer structure are called as a, b, c, d, e, f, and g. The pressure levels that define each of these layers are named as $p_{\text {lay1 }}$ and $p_{\text {lay } 2}$ for the upper and bottom limit of the layer, respectively.

structure with aerosols and gas uniformly mixed with a clear gas layer from the top of the atmosphere to the $10 \mathrm{mbar}$ level (see Fig. 13). The calculated results at this latitude seem to indicate that stratospheric particles (from 0.4 mbar to $\sim 80$ mbar) are quite different from tropospheric parti- cles (between 140 and 400 mbar) with a transition region located between 80 and 240 mbar. The value of the imaginary part of the refractive index of the upper stratospheric particles at UV wavelengths is much larger $(0.09-0.02)$ than the values at the deeper tropospheric regions (0.009) (see 
Table 6). The real part of the refractive index had also to be changed at different atmospheric levels in order to get a good match with the observational data. This seems to support the idea that tropospheric particles at the polar regions are produced by a different mechanism than stratospheric particles as suggested by Karkoschka and Tomasko (1993). The haze optical depth at stratospheric levels is larger at the polar region than at other latitudes on the planet (see Tables 5 and 6). West et al. (1983a, 1983b), also found by analysis of the Voyager photopolarimeter measurements a significant optical depth of UV absorbers at the north polar region. Aerosol particles in the polar regions are probably created by different mechanisms that in other regions. Besides photochemical processes, the origin of these aerosol are probably related to energetic particle bombardment at high latitudes. Pryor and Hord (1991) suggested several sources of production of aerosols, the most likely being hydrocarbon aerosol formation initiated from aurorally produced $\mathrm{H}_{2}^{+}$ionization. Moreover, Gérard et al. (1995), proved that auroral production of hydrocarbon aerosols is a possible source for the high latitude haze. In addition, as pointed out by these authors, the latitudinal and vertical temperature gradient associated with the auroral heating rate over the solar heating can lead to a combined effect of vertical and horizontal transport in a similar way to the jovian aurora. This could explain the differences in the vertical structure of clouds when comparing with the equator and mid-latitudes.

As far as the other studied latitudes is concerned, our images clearly show the southern (spring) hemisphere brighter in the methane image than the northern (fall) hemisphere (see Fig. 2). According to the results of our model, the north/south asymmetries in the reflectivity data are due to tropospheric particles located at higher levels in the southern hemisphere. The upper tropospheric limit, $p_{3}$, is located at 87 mbar at $-50^{\circ}$ while at $+50^{\circ}$ it is found at 140 mbar. In the same way, $p_{3}$ is located at $60 \mathrm{mbar}$ at $30^{\circ}$ south being located and 90 mbar at $30^{\circ}$ north. The upper tropospheric haze boundary, presents strong latitudinal variations too. In order to reproduce the low observational reflectivity values at $893 \mathrm{~nm}$ together with the relatively high ultraviolet reflectivity values at $+50^{\circ}$ (see Fig. 2), the upper limit of the tropospheric haze, as well as the stratospheric haze, has to be located at deeper atmospheric levels than at the other studied latitudes. At $+50^{\circ}$ latitude, $p_{3}$ has a value of $140 \mathrm{mbar}$ whereas it varies between 60 and 90 mbar at other latitudes (see Fig. 13 and Table 3).

The lower boundary of the tropospheric cloud, $p_{2}$, presents the same value at the two studied mid-latitudes at the southern hemisphere. It extends down to 400 mbar at $-50^{\circ}$ and $-30^{\circ}$, with particles located at higher altitudes at $-10^{\circ}$ (180 mbar). In contrast, $p_{2}$ varies at the two latitudes studied at the northern hemisphere from $600 \mathrm{mbar}$ at $+30^{\circ}$, to 350 mbar at $+50^{\circ}$.

The calculated pressure, at the bottom of the tropospheric haze, $p_{2}$, agrees well with the results found by Stam et al. (2001), based on spectra obtained in August 1995. At that date, it was late summer in the northern hemisphere, the northern fall would start 3 months later, on November 19, 1995. These authors also obtained the upper tropospheric haze located at higher levels at the southern hemisphere, finding the same values of $p_{2}$ at $-30^{\circ}$ and $+30^{\circ}$ as derived in this work. Stam et al. (2001) also found the tropospheric haze higher in the equatorial regions than at mid-latitudes. The upper limit of the tropospheric haze, $p_{3}$, was located at slightly higher levels in this work than in Stam et al. (2001) (80 mbar at southern mid-latitudes and 100 mbar at northern mid-latitudes). However, they also found the same latitudinal variation, with $p_{3}$ approximately constant in the southern hemisphere, increasing with latitude in the northern hemisphere. Interestingly, other authors (see, e.g., West et al., 1982) found a reversed asymmetry, i.e., the northern hemisphere was brighter in the methane band when the planet was close to southern fall equinox (more than half Saturn year earlier). Barnet et al. (1992b), also found summertime enhancement in the blue reflectivity in the northern hemisphere. West et al. (1982), suggested cloudy material located at higher altitudes in the bright hemisphere to explain the observed asymmetry in the methane band observations. The fact that in our observations and the Stam et al. (2001) observations the brightest hemisphere is the southern hemisphere supports the theory of a possible relation between the north-south asymmetries and seasonal changes. This relation between the north/south asymmetry and seasonal effects was also suggested by other authors (see, e.g., West et al., 1982; Stam et al., 2001). Bézard et al. (1994) modeled the seasonal variability of temperature in the troposphere of Saturn. These authors conclude that the observed hemispherical asymmetry of the temperature profiles originates in the seasonally variable insolation. As suggested by Trafton (1985), cooler temperatures in the northern hemisphere would saturate $\mathrm{NH}_{3}$ gas, forming haze particles in addition to the haze formed from upward eddy diffusion of $\mathrm{NH}_{3}$. Indeed, we have also derived larger tropospheric optical depths in the northern hemisphere when comparing the results at $-50^{\circ}$ with those at $+50^{\circ}$, and the results at $-30^{\circ}$ with those at $+30^{\circ}$ (see Table 5). The largest optical depth at this atmospheric level has been found at $-10^{\circ}$ (see Table 5). Strong latitudinal variations of tropospheric optical depths, increasing from mid-latitudes toward equatorial regions, were also found by Karkoschka and Tomasko (1993) and Ortiz et al. (1996).

The stratospheric haze extends to high atmospheric levels as suggested by Karkoschka and Tomasko (1993) (see Fig. 13). As mentioned before, by inspection of Fig. 2, reflectivity data at $+50^{\circ}$ seem to indicate the existence of a relatively high amount of UV absorbers but located at deeper layers than at other latitudes, as the very low reflectivity values at the methane absorption band show. In this case, stratospheric particles are located between 30 mbar and 6 mbar whereas at the other studied latitudes are located at higher atmospheric levels (between 1 mbar and 10 mbar at 
$-30^{\circ}$, and $+30^{\circ}$; between 1 mbar and $12 \mathrm{mbar}$ at $-50^{\circ}$, and between 20 and 2 mbar at $-10^{\circ}$ (see Fig. 13).

There are some discrepancies between the values presented in this work for $p_{4}$ and those obtained by Ortiz et al. (1996) and Stam et al. (2001). We could not find a good fit by using values of $p_{4}$ and $p_{5}$ within the range mentioned in Section 3. Ortiz et al. (1996) reported values for $p_{4}$ between 40 and 60 mbar, independent of the latitude, whereas Stam et al. (2001), presented values increasing with latitude from 20 mbar near the equator to 40 mbar at midlatitudes. We have also found lower values of $p_{4}$ than those obtained by Ortiz et al. (1996). In our case, the stratospheric haze was placed between 1 and $\sim 10$ mbar at the southern hemisphere and $+30^{\circ}$, being situated much deeper (between 6 and 30 mbar) at $+50^{\circ}$. Stratospheric optical depths are relatively small at all wavelengths (see Table 5), increasing toward equatorial latitudes. This atmospheric haze also presents north/south differences when comparing calculated values at both hemispheres, with the largest optical depths at the northern (fall) hemisphere. This tendency was also found by Stam et al. (2001).

The results of this work seem to support the idea of a relation between the north/south asymmetry and seasonal effects. Our results are in agreement with the results obtained by other authors. This agreement is remarkable considering the very different techniques used in the different works. As future work, we plan to study the Saturn's vertical structure of hazes at different time periods in the same wavelengths described here in order to compare the results of the same method when applied to different saturnian seasons.

\section{Acknowledgments}

The authors are grateful to two anonymous referees for their valuable comments and suggestions. Fruitful discussions with D.M. Stam on a previous version of this manuscript are gratefully acknowledge. This work is based on observations with the NASA/ESA Hubble Space Telescope, obtained at the Space Telescope Science Institute (STScI), which is operated by the Aura, Inc. for NASA. This work was supported by Contracts PNE-001/2000-C-01 and AYA2001-1177. J.C. Gérard and D. Grodent acknowledge support from the Belgiam Fund for Scientific Research and the PRODEX program of ESA.

\section{References}

Acarreta, J.R., Sanchez-Lavega, A., 1999. Vertical cloud structure in Saturn's 1990 equatorial storm. Icarus 137, 24-33.

Barnet, C.D., Beebe, R.F., Conrath, B.J., 1992a. A seasonal radiativedynamic model of Saturn's troposphere. Icarus 98, 94-107.

Barnet, C.D., Westphal, J.A., Beebe, R.F., Huber, L.F., 1992b. Hubble Space Telescope observations of the 1990 equatorial disturbance on Saturn-zonal winds and central meridian albedos. Icarus 100, 499511.
Bézard, B., Gautier, D., Conrath, B., 1994. A seasonal model of the saturnian upper troposphere: comparison with Voyager infrared measurements. Icarus 60, 274-288.

Conrath, B.J., Gautier, D., 2000. Saturn helium abundance: a reanalysis of Voyager measurements. Icarus 144, 124-134.

Danielson, R.E., Tomasko, M.G., 1969. A two-layer model of the jovian clouds. J. Atmos. Sci. 26, 889-897.

Gérard, J.C., Dols, V., Grodent, D., Waite, J.H., Gladstone, G.R., Prange, R., 1995. Simultaneous observations of the saturnian aurora and polar haze with the HST/FOC. Geophys. Res. Lett. 22, 2685-2689.

Holtzman, J., Burrows, J.C., Casertano, S., Hester, J., Trauger, J.T., Watson, A.M., Worthey, G., 1995. The photometric performance and calibration of WFPC2. Publ. Astron. Soc. Pacific 107, 1065-1093.

Hubbard, W.B., Marley, M.S., 1989. Optimized Jupiter, Saturn, and Uranus interior models. Icarus 78, 102-118.

Karkoschka, E., 1994. Spectrophotometry of the jovian planets and Titan at 300- to 1000-nm wavelength: the methane spectrum. Icarus 111,174 192.

Karkoschka, E., 1998a. WFPC2 Photometry for the Solar System. Space Telescope Science Institute, Baltimore.

Karkoschka, E., 1998b. Methane, ammonia, and temperature measurements of the jovian planets and Titan from CCD spectrophotometry. Icarus 133, 134-146.

Karkoschka, E., Tomasko, M.G., 1992. Saturn's upper troposphere 19861989. Icarus 97, 161-181.

Karkoschka, E., Tomasko, M.G., 1993. Saturn's upper atmospheric hazes observed by the Hubble Space Telescope. Icarus 106, 428-441.

Lindal, G.F., Sweetnam, D.N., Eshleman, V.R., 1985. The atmosphere of Saturn-an analysis of the Voyager radio occultation measurements. Astron. J. 90, 1136-1146.

Molina, A., Moreno, F., Muñoz, O., 1997. Aerosol debris in the core and crescent-shaped regions of Comet P/Shoemaker-Levy $9 \mathrm{H}$ and $\mathrm{G}$ fragment impact sites on Jupiter. Icarus 127, 213-220.

Moreno, F., Muñoz, O., Molina, A., López-Moreno, J.J., Ortiz, J.L., Rodrí guez, J., López-Jimenez, A., Girela, F., Larson, S.M., Campins, H., 1995. Physical properties of the aerosol debris generated by the impact of fragment H of Comet P/Shoemaker-Levy 9 on Jupiter. Geophys. Res. Lett. 22, 1609-1612.

Muñoz, O., Moreno, F., Molina, A., Ortiz, J.L., 1999. A comparison of the structure of the aerosol layers in the great red SPOT of Jupiter and its surroundings before and after the 1993 SEB disturbance. Astron. Astrophys. 344, 355-361.

O'Brien, J.J., Cao, H., 2002. Absorption spectra and absorption coefficients for methane in the 7500-940 $\mathrm{nm}$ region obtained by intracavity laser spectroscopy. J. Quant. Spectrosc. Radiat. Transfer 75, 323-350.

Ortiz, J.L., Moreno, F., Molina, A., 1995. Saturn 1991-1993: reflectivities and limb-darkening coefficients at methane bands and nearby continuatemporal changes. Icarus 117, 328-344.

Ortiz, J.L., Moreno, F., Molina, A., 1996. Saturn 1991-1993: clouds and hazes. Icarus 119, 53-66.

Owen, T., 1969. The spectra of Jupiter and Saturn in the photographic infrared. Icarus 10, 355-364.

Prinn, R.G., Larson, H.P., Caldwell, J.J., Gautier, D., 1984. Composition and chemistry of Saturn's atmosphere. In: Gehrels, T., Matthews, M.S. (Eds.), Saturn. Univ. of Arizona Press, Tucson, pp. 88-149.

Pryor, W.R., Hord, C.W., 1991. A study of photopolarimeter system UV absorption data on Jupiter, Saturn, Uranus, and Neptune-implications for auroral haze formation. Icarus 91, 161-172.

Stam, D.M., Banfield, D., Gierash, P.J., Nicholson, P.D., 2001. Near-IR spectrophotometry of saturnian aerosols-meridional and vertical distribution. Icarus 152, 407-422.

Stamnes, K., Tsay, S.C., Wiscombe, W., Jayaweera, K., 1988. Numerically stable algorithm for discrete-ordinate-method radiative transfer in multiple scattering and emitting layered media. Appl. Optics 27, 25022509.

Tomasko, M.G., Doose, L.R., 1984. Polarimetry and photometry of Saturn from Pioneer 11 observations and constraints on the distribution and properties of cloud and aerosol particles. Icarus 58, 1-34. 
Tomasko, M.G., West, R.A., Orton, G.S., Teifel, V.G., 1984. Clouds and aerosols in Saturn's atmosphere. In: Gehrels, T., Matthews, M.S (Eds.), Saturn. Univ. of Arizona Press, Tucson, pp. 150-194.

Trafton, L., 1985. Long-term changes in Saturn's troposphere. Icarus 63, 374-405.

Van de Hulst, H.C., 1980. Multiple Light Scattering: Tables, Formulas and Applications. Academic Press, New York.

Wagener, R., Caldwell, J., 1988. Strong north/south asymmetry in the jovian stratosphere. Icarus $74,141-152$.

Wallace, L., Caldwell, J.J., Savage, B.D., 1972. Ultraviolet photometry from the orbiting astronomical observatory III. Observations of Venus, Mars, Jupiter, and Saturn longward of 2000 A. Astrophys. J. 172, 755769.
West, R.A., Tomasko, M.G., Smith, B.A., Wijesinghe, M.P., Doose, L.R., Reitsema, H.J., Larson, S.M., 1982. Spatially resolved methane band photometry of Saturn. I. Absolute reflectivity and center-to-limb variations in the 6190-, 7250-, and 8900- $\AA$ bands. Icarus 51, 51-64.

West, R.A., Sato, M., Hart, H., Lane, A.L., Hord, C.W., Simmons, K.E., Esposito, L.W., Coffeen, D.L., Pomphrey, R.B., 1983a. Photometry and polarimetry of Saturn at 2640 and 7500 A. J. Geophys. Res. 88, 86798697.

West, R.A., Hord, C.W., Simmons, K.E., Hart, H., Esposito, L.W., Lane, A.L., Pomphrey, R.B., Morris, R.B., Sato, M., Coffeen, D., 1983b. Voyager photopolarimeter observations of Saturn and Titan. Adv. Space Res. 3, 45-48. 